\title{
Snf1p/Hxk2p/Mig1p pathway regulates exponential growth, mitochondrial respiration, and hexose transporters transcription in Saccharomyces cerevisiae
}

Andres Carrillo-Garmendia ${ }^{1}$, Cecilia Martinez-Ortiz ${ }^{1}$, Jairo Getzemani Martinez-Garfias ${ }^{2}$, Juan Carlos González-Hernández ${ }^{3}$, Gerardo M. Nava ${ }^{4}$, Minerva Ramos-Gomez ${ }^{4}$, Miguel David DufooHurtado $^{1}$ and Luis Alberto Madrigal-Perez ${ }^{2 *}$

From the; ${ }^{1}$ Universidad Politécnica de Guanajuato, Av. Universidad Sur \#1001, Cortazar, Guanajuato, 38496, México; ${ }^{2}$ Tecnológico Nacional de México/ Instituto Tecnológico Superior de Ciudad Hidalgo, Av. Ing. Carlos Rojas Gutiérrez \#2120, Ciudad Hidalgo, Michoacán, 61100, México; ${ }^{3}$ Tecnológico Nacional de México/ Instituto Tecnológico de Morelia, Av. Tecnológico de Morelia, Morelia, Michoacán, 58030, México; ${ }^{4}$ Universidad Autónoma de Querétaro, Cerro de las Campanas, Santiago de Querétaro, Qro, 76010, México.

* To whom correspondence should be addressed: Luis Alberto Madrigal-Perez: Tecnológico Nacional de México/ Instituto Tecnológico Superior de Ciudad Hidalgo, Av. Ing. Carlos Rojas Gutiérrez \#2120, Ciudad Hidalgo, Michoacán, 61100, México. lmadrigal@cdhidalgo.tecnm.mx. Phone: +52 (786) 154-9000. Fax: +52 (786) 154-9000.

Running title: Energy metabolism affected by Snf1p/Hxk2p/Mig1p pathway

Keywords: AMP-activated kinase (AMPK); cell growth; Saccharomyces cerevisiae; mitochondrial respiration; mitochondrial metabolism; cell signaling. 


\section{Abstract}

53 The Crabtree effect occurs under high-glucose concentrations and is characterized by the increase of the growth and a decrease in mitochondrial respiration of yeasts. Regulation of the Crabtree effect could enhance ethanol production with biotechnological purposes and a better understanding of the etiology of cancer due to its similitude with the Warburg effect. Nonetheless, the conclusive molecular mechanism of the Crabtree effect is still on debate. The pathway Snf1p/Hxk2p/Mig1p has been linked with the transcriptional regulation of the hexose transporters and has also been identified in the modulation of phenotypes related to the Crabtree effect. Nevertheless, it has not been directly identified the genetic regulation of the hexose transporters with modulation of the Crabtree effect phenotypes by Snf1p/Hxk2p/Mig1p pathway. In this sense, we provide evidence that the deletion of the SNF1 and $H X K 2$ genes affects the exponential growth, mitochondrial respiration, and the transcription of hexose transporters in a glucose-dependent manner in Saccharomyces cerevisiae. The Vmax of the main hexose transporters transcribed showed a positive correlation with the exponential growth and a negative correlation with the mitochondrial respiration. Transcription of the gene HXT2 was the most affected by the deletion of the pathway SNF1/HXK2/MIG1. Deletion of the orthologous genes SNF1 and HXK2 in the Crabtree negative yeast, $K$. marxianus, has a differential effect in exponential growth and mitochondrial respiration in comparison with $S$. cerevisiae. Overall, these results indicate that the SNF1/HXK2/MIG1 pathway transcriptionally regulates the hexose transporters having an influence in the exponential growth and mitochondrial respiration in a glucose-dependent manner. 


\section{Introduction}

104 The metabolic flexibility of organisms to form ATP under different environmental circumstances is a valuable phenotype to perpetuating its species. In the case of Saccharomyces cerevisiae, it has evolved an intricate signaling pathway to adapt its metabolism depending on the carbohydrate accessibility to form ATP by fermentation (substrate-level phosphorylation) or by mitochondrial respiration (oxidative phosphorylation) [1,2]. Sugar concentration above $0.8 \mathrm{mM}$ activates a metabolic pathway called glucose repression that reshapes the metabolism to produce ATP mainly by fermentation; accompanied by an increase in the glycolytic flux, decreasing the mitochondrial respiration and increasing the fermentation metabolites, altogether this phenotype is called Crabtree effect. However, the molecular basis behind the Crabtree effect is still unknown. Some hypotheses point out that the glycolysis pathway is the key step during the Crabtree effect. For example, the increase of the glycolysis-derived hexose phosphate, fructose1,6-bisphosphate inhibits the respiratory chain in isolated mitochondria of $S$. cerevisiae [3]. Additionally, glucose supplementation in AS-30D hepatoma cells decreases intracellular phosphate $\left(\mathrm{P}_{\mathrm{i}}\right)$ and increases the AMP, glucose-6-phosphate, fructose-6-phosphate, and fructose1,6-bisphosphate [4]. Interestingly, when isolated mitochondria were incubated with low $\mathrm{P}_{\mathrm{i}}(0.6$ $\mathrm{mM}$ ), inhibition of the mitochondrial respiration was also observed [4]. Besides, it has been shown that the augmenting of glucose concentration in S. cerevisiae increases the glycolytic flux accompanied by inhibition of mitochondrial respiration [5]. Altogether, these data indicate that an increase in the glycolytic pathway flux is important to unleash the Crabtree effect. Nonetheless, which signal/pathway regulates this increase in glycolytic flux is still unknown. One hypothesis suggests that hexose transporters could regulate the glycolytic flux. For instance, in a $S$. cerevisiae strain in which all the hexose transporters were deleted, the expression of a lowaffinity transporter Hxtlp ( $K m$ around $90 \mathrm{mM}$ and Vmax $\sim 690 \mathrm{nmol} \mathrm{min}^{-1} \mathrm{mg}^{-1}$ [6]) have a major glycolytic flux than the expression of the high-affinity transporter Hxt7p $(\mathrm{Km} \sim 1 \mathrm{mM}$ and Vmax $\sim 186 \mathrm{nmol} \mathrm{min}^{-1} \mathrm{mg}^{-1}$ [6]) [7]. Interestingly, the diminution in the glycolytic flux resulted in a decrease in the ethanol accumulation accompanied by an increase in the mitochondrial respiration [7]. It has been proposed that the glucose uptake has a linear correlation with the ethanol production in S. cerevisiae, which implies that hexose transporters could play an important role in the switch between fermentation and mitochondrial respiration [8]. The hexose transporters have different biochemical properties. Thereby, the expression of the hexoses transporter occurs under different sugar concentrations and fits with each environmental situation. Nevertheless, the signaling pathway that modulates those changes in the hexose transporters expression is not well known.

The protein Snf1p is AMP-activated protein kinase (orthologous to the mammalian AMPK) that responds to energetic changes mainly through the ADP/ATP ratio in $S$. cerevisiae [9]. Interestingly, the deletion of the gene $S N F 1$ causes a severe metabolic disruption in the response to glucose concentrations [10-12]. For example, SNF1 deletion reverses the mitochondrial respiration inhibition in high-glucose concentrations [10]. Snf1p regulates by phosphorylation the translocation of the nucleus of two important regulators of glucose repression: Hxk2p [13] and Mig1p [14]. Hxk2p has a dual function, as catalytic in the first reaction of glycolysis pathway and as a transcriptional regulator. $\mathrm{Hx} 2 \mathrm{p}$ also serves as an intracellular glucose sensor changing its conformation to interact with Mig1p under high-glucose concentrations to repress SUC2 promoter [15]. Even, Hxk2p is necessary for the inhibition of the Mig1p by Snf1p, when both form part of the repressor complex [13]. Mig1p is a $\mathrm{Cys}_{2}-\mathrm{His}_{2}$ zinc finger protein that binds to glucoserepressible genes and its localization in the nucleus is dependent on the glucose concentration. Interestingly, Mig1p regulates transcriptionally the promoters of the genes HXT2 and HXT4 151 preventing its transcription at $4 \%$ glucose [16]. Indeed, the deletion of the gene MIG1 increases 152 the transcription of the hexose transporters HXT3, HXT4, HXT6, and HXT7 at $4 \%$ glucose; while 

transcription of the gene $H X T 4$ was augmented in the mig $1 \triangle$ strain at $2 \%$ and $10 \%$ glucose [18].

For this reason, the aim of this study was to elucidate if the pathway Snf1p/Mig1p/Hxk2p is responsible for the regulation of transcription of hexose transporters in a glucose-dependent manner and its relationship with exponential growth and mitochondrial respiration in yeasts. The data provided in this paper indicate that $S$. cerevisiae regulates the transcription of the hexose transporters in a glucose-dependent manner by the pathway Snf1p/Mig1p/Hxk2p. Specifically, the transcription of the high-affinity $H X T 2$ was several affected by the deletion of the genes SNF1, HXK2, and MIG1. Importantly, we also found that exponential growth correlates positively with the Vmax of the mainly transcribed hexose transporters and correlates negatively with the basal respiration. These data indicate that hexose transporters might have a relevant impact on exponential growth and mitochondrial respiration and are transcriptionally regulated by the pathway Snf1p/Mig1p/Hxk2p.

\section{Material and methods}

\section{Saccharomyces cerevisiae strains}

For performing this study, it was used the S. cerevisiae strain BY4741 (MATa; ura3 40 ; leu2A0;

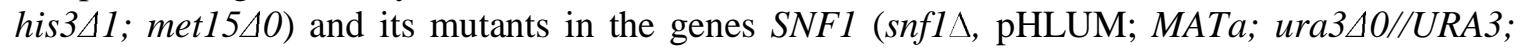
leu2A0//LEU2; his3A0//HIS3; met15

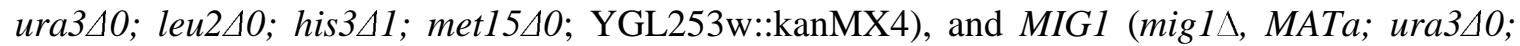
leu2A0; his3 11 ; met15 0 ; YGL035c::kanMX4). Also some experiments were performed with the

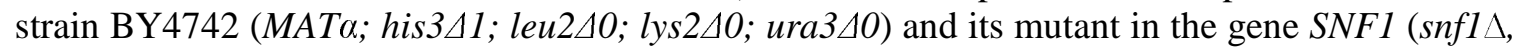

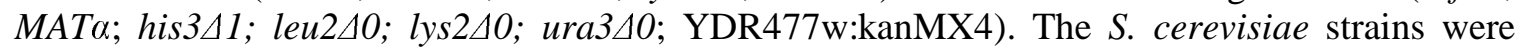
maintained in YPD medium (1\% yeast extract, $2 \%$ casein peptone, and supplemented with $2 \%$ glucose), deletant strains were supplemented with geneticin (G-418 disulfate salt solution, SigmaAldrich) at a final concentration of $200 \mu \mathrm{g} / \mathrm{mL}$.

\section{Kluyveromyces marxianus deleted strains}

K. marxianus L-2029 strain was used to obtain deletions in the genes SNF1 and RAG5 orthologous to the $S$. cerevisiae genes $S N F 1$ and $H X K 2$, respectively. The genes $S N F 1$ and RAG5 were interrupted by homologous recombination using a PCR-based gene deletion strategy [19]. First, the kanMX4 (to confer resistance to geneticin) gene was amplified with the primers: forward 5-ATGCGTACGCTGCAGGTCGAC-3 ${ }^{\circ}$ and reverse 5TCAATCGATGAATTCGAGCTCG-3` [20]. In the second instance, a 45mers primers flanking upstream and downstream the open reading frame (ORF) of the interest gene (SNF1 and RAG5) and with homology to the kanMX4 were used. SNF1 primers: forward 5AAAAAGGGGAAACAACAGAGAGATACAACAATTAAATTGGACATG -3` and reverse 5 TTACGTAATGAACTAACCGGTATGGAAGAGAAACACAAAAACTCA-3`. RAG5 primers: forward 5`- GGTTTACGCCTCCAATTATTACAAACACACACACTTACAAAAATG-3` and reverse 5- CTTCGATACCAACAGACTTACCGGCAGCCAATCTCTTTTGTGTCA-3`. The PCR products were inserted in $K$. marxianus using lithium acetate transformation protocol described by Gietz, St Jean, Woods and Schiestl [21] with some modifications. Briefly, $K$. marxianus was grown in a $250 \mathrm{~mL}$ shake-flask with $50 \mathrm{~mL}$ of YPD media at $30^{\circ} \mathrm{C}$ and $250 \mathrm{rpm}$ until it reaches the mid-log phase (O.D.600. 0.6). Cells were harvest at $4000 \mathrm{x} g$ for 2 minutes at room temperature and washed two times with distilled water. Afterwards, cells were washing with TE/LiAc (10x TE; 0.1 M Tris-HCl, 0.01 M EDTA, pH 7.5; 10x lithium acetate (LiAc); $1 \mathrm{M}$ LiAc $\mathrm{pH} 7.5$, adjusted with diluted acetic acid) and resuspended in $1 \mathrm{~mL} \mathrm{1x} \mathrm{TE/LiAc.} \mathrm{Cells}$ transformation was carried out in a $1.5 \mathrm{~mL}$ microtube with $50 \mu \mathrm{L}$ cells, $12 \mu \mathrm{L}$ PCR product, 50 $\mu \mathrm{g}$ salmon sperm DNA, and $300 \mu \mathrm{L}$ PEG 4000 solution (40\% de PEG 4000, 1x TE, 1x LiAc). Microtube was incubated at $30{ }^{\circ} \mathrm{C}$ for 30 minutes and $100 \mathrm{rpm}$, follow by a termic shock in a 
water bath at $42{ }^{\circ} \mathrm{C}$ for 15 minutes, cells were harvest at $13000 \mathrm{x} g$ for 5 seconds and resuspended in $1 \mathrm{~mL} 1 \mathrm{x}$ TE solution. Finally, transformant cells were selected in a YPD plate with $200 \mu \mathrm{g} / \mathrm{mL}$ geneticin (G-418, Sigma-Aldrich).

\section{6}

207

\section{Over-expression of the gene $H X K 2$}

The pYES2.1 TOPO TA yeast expression kit (Invitrogen) was used to over-express the gene $H X K 2$. Primers to amplify the ORF of the gene $H X K 2$ were designed to obtain the ORF without stop codon. HXK2 primers: forward 5'-ATGGTTCATTTAGGTCCAAAAAAACC-3' and reverse 5'-AGCACCGATGATACCAACGGAC-3'. PCR reactions contained: $1 \mu \mathrm{L}$ primers at $10 \mathrm{mM}, 1$ $\mu \mathrm{L}$ dNTP mix at $10 \mathrm{mM}, 5 \mu \mathrm{L}$ Dream Taq 10x buffer, $0.25 \mu \mathrm{L}$ Dream Taq, $100 \mathrm{ng}$ of $S$. cerevisiae genomic DNA, and $40.75 \mu \mathrm{L}$ nuclease-free water. Amplicons were purified using PureLink PCR purification kit (Invitrogen). The plasmid constructs were cloned into Escherichia coli $\mathrm{TOP} 10\left(\mathrm{~F}^{-}\right.$mcrA $\Delta($ mrr-hsdRMS-mcrBC) $\varphi 80$ lacZ $\Delta \mathrm{M} 15 \Delta l a c \mathrm{X} 74$ recA1 araD139 $\Delta$ (araleu)7697 galU gal $\mathrm{K} \lambda^{-} \operatorname{rpsL}\left(\mathrm{Str}^{\mathrm{R}}\right)$ end $\mathrm{A} 1$ nup $\left.\mathrm{G}\right)$ by chemical transformation, which served as a bacterial host for the recombinant plasmid. Finally, yeast strains were transformed using LiAc method [22].

\section{Growth kinetics}

The growth kinetics were carried out as described by Olivares-Marin, Madrigal-Perez, CanizalGarcia, Garcia-Almendarez, Gonzalez-Hernandez and Regalado-Gonzalez [5]. Assay of overexpression in $S$. cerevisiae come from pre-inoculum grown with SC media $(6.7 \mathrm{~g} / \mathrm{L}$ yeast nitrogen base without amino acids, $2 \mathrm{~g} / \mathrm{L}$ yeast synthetic drop-out media supplements without uracil, $0.05 \mathrm{~g} / \mathrm{L}$ uracil, glucose according to the experiment), while growth kinetics of Kluyveromyces marxianus and S. cerevisiae were made with pre-inoculums grown in YPD media. Growth kinetics were started at O.D.600 $\bullet 0.01$ in a 96-well plate that contained $200 \mu \mathrm{L}$ of YPD media supplemented with $0.005 \%$ or $5 \%$ glucose. The 96 -well plate was incubated at $30{ }^{\circ} \mathrm{C}$ for 15 hours with constant shaking. The growth was monitored measuring the O.D. 600 each hour using a microplate reader (Multiskan Go; Thermo-Scientific). Data were analyzed with the statistical package GraphPad Prism 6.00 for Macintosh (GraphPad Software) fitting the growth kinetics with the exponential growth equation to obtain the specific growth rate $(\mu)$.

\section{Mitochondrial respiration analyses}

The in situ mitochondrial respiration was performed as described by Tello-Padilla, PerezGonzalez, Canizal-Garcia, Gonzalez-Hernandez, Cortes-Rojo, Olivares-Marin and MadrigalPerez [23]. The pre-inoculum cultures were made in SC medium supplemented with $2 \%$ glucose and were used to select the recombinant strains (without uracil) and deleted strains (with geneticin). Then, the strains were cultured in $250 \mathrm{~mL}$ shake-flasks with $50 \mathrm{~mL}$ of YPD medium supplemented with $0.005 \%$ or $5 \%$ glucose at $30{ }^{\circ} \mathrm{C}$ and $250 \mathrm{rpm}$ until the mid-log phase $\left(\mathrm{OD}_{600} \sim 0.5\right)$ was reached. Next, cells were harvested at $4000 \mathrm{x} g$ for 5 minutes and washed three times with deionized water, and resuspended in a 1:1 ratio $(w / v)$. Immediately, cells were ready to measure the oxygen consumption polarographically with a Clark type electrode connected to a YSI5300A monitor (Yellow Springs, OH, USA) and a computer for data acquisition. In the polarograph chamber were added: $125 \mathrm{mg}$ of cells (wet weight), $5 \mathrm{~mL}$ of MES-TEA buffer (10 $\mathrm{mM}$ morphoethanolsulfonic acid, $\mathrm{pH} 6.0$ with triethanolamine) and glucose $(10 \mathrm{mM})$; the cells were maintained with constant agitation (basal respiration). Afterward, $0.015 \mathrm{mM}$ carbonylcyanide- $p$-trichlorophenylhydrazone (CCCP) was added to the chamber to stimulate the uncoupled state (maximum respiration) and oxygen consumption was recorded for 3 minutes. Finally, inhibitors of the electron transport chain (ETC) were added: thenoyltrifluoroacetone (TTFA, $1 \mathrm{mM}$ ), antimycin A (AA), and $0.75 \mathrm{mM} \mathrm{KCN} \mathrm{(non-mitochondrial} \mathrm{respiration),} \mathrm{each}$ inhibitor was left for 3 minutes. The results were analyzed using the statistical package GraphPad Prism 6.00 for Macintosh (GraphPad Software). 


\section{RT-qPCR}

The strains (BY4741 genetic background) were grown in $25 \mathrm{~mL}$ shake-flask with $5 \mathrm{~mL}$ of SC medium supplemented with $0.005 \%$ or $5 \%$ glucose until the mid-log phase $\left(\mathrm{OD}_{600} \sim 0.5\right)$ was reached. The second experiment was made with the strains with a genetic background BY4742, which were grown in $25 \mathrm{~mL}$ shake-flask with $5 \mathrm{~mL}$ of YPD medium supplemented with $0.01 \%$ or $5 \%$ glucose until the mid-log phase $\left(\mathrm{OD}_{600} \sim 0.5\right)$ was reached. Cells were harvested at $13000 \times \mathrm{g}$ for 3 minutes. TRIzol reagent (Ambion, Life Technologies) was used to isolated the total RNA following the manufacturer`s instructions. The RNA pellet was resuspended in $50 \mu \mathrm{L}$ diethylpyrocarbonate water (DEPC), adding recombinant DNAase I, RNAse free (Roche Diagnostics $\mathrm{GmbH}$ ). The integrity of the RNA was verified with an electrophoretic analysis, as well as the concentration and quality of the RNA using a $\mu$ Drop plate in the Multiskan Go spectrophotometer (Thermo-Scientific).

To carry out the qPCR the cDNA synthesis was performed using $2.5 \mu \mathrm{g}$ of total RNA, $200 \mathrm{ng}$ of random hexamer primers, and $0.65 \mathrm{mM}$ of dNTPs. The reaction mix was incubated at $65{ }^{\circ} \mathrm{C}$ for 5 minutes. Then, to reaction were added $4 \mu \mathrm{L}$ of $5 \mathrm{X}$ buffer of RevertAid and $2 \mu \mathrm{L}$ of DTT $0.1 \mathrm{M}$ and incubated at $37^{\circ} \mathrm{C}$ for 2 minutes. Finally, $200 \mathrm{U}$ of the RevertAid RT was added, followed by two periods of incubation, 10 minutes at room temperature and at $37{ }^{\circ} \mathrm{C}$ for 50 minutes. Each qPCR reaction had a final volume of $10 \mu \mathrm{L}, 1 \mu \mathrm{L}$ of cDNA, $5 \mu \mathrm{L}$ of SYBR Select Master Mix (Applied Biosystems), $0.4 \mu \mathrm{L}$ of each primer and $3.2 \mu \mathrm{L}$ of DEPC water were added. Subsequently, the PCR was performed in a Rotor-Gene Q thermocycler (Qiagen). Absolute quantification was used to quantify the transcription using as a reference gene $U B C 6$ (ubiquitinconjugation-6) (Table 1). Conditions of the PCR were: activation of UDG at $50{ }^{\circ} \mathrm{C}$ for 2 minutes, followed by the activation of AmpliTaq-DNA at $95{ }^{\circ} \mathrm{C}$ for 2 minutes with 35 cycles of denaturation at $94{ }^{\circ} \mathrm{C}$ for 15 seconds. The alignment temperature of primers HXT1, HXT3, HXT4, HXT6, HXT7, and $H X T 10$ was $50{ }^{\circ} \mathrm{C}$, for $H X T 2$ was $62{ }^{\circ} \mathrm{C}$, and for $U B C 6$ was $51{ }^{\circ} \mathrm{C}$ for 30 seconds, and the extension temperature of $72{ }^{\circ} \mathrm{C}$ for 1 minute, followed by 1 cycle at $72{ }^{\circ} \mathrm{C}$ for 7 minutes.

\section{Statistical analyses \\ Data were obtained from at least three independent experiments and the mean \pm standard deviation was graphed. Means were compared using one-way ANOVA followed by a Dunnett multiple comparisons to analyze differences in exponential growth and mitochondrial respiration. Differences between means in hexose transporters transcription were tested using a two-tailed unpaired $t$-test. Pearson's correlation analysis was carried to evaluate the relationship between exponential growth/mitochondrial respiration and Vmax. Statistical analyses were computed in the software GraphPad Prism 6.00 for Macintosh (GraphPad Software).}

Principal component analysis (PCA) plot was generated using ClustVis: (http://biit.cs.ut.ee/clustvis) utilizing a probabilistic PCA method without row scaling and employing the function $(\operatorname{In} x+1)$ for transformation [24].

\section{Results}

\section{Effect of $H X K 2$ and $S N F 1$ deletion on the growth of $S$. cerevisiae}

The transduction signal of the $\mathrm{Snf} 1 \mathrm{p} / \mathrm{Hxk} 2 \mathrm{p}$ pathway responds primarily to glucose, impacting in the form that cells produce ATP. Exponential growth is tightly coupled to the form to obtain ATP in $S$. cerevisiae, showing higher rates of growth when having a fermentative growth in comparison when having respiratory growth [5]. We hypothesize that hexose transporters are the principals responsible for reshaping the $S$. cerevisiae cells to switch between mitochondrial 305 respiration and fermentation, regulating the glycolytic flux. Taken those ideas into account, we 
propose that $S$. cerevisiae exponential growth has a correlation with the biochemical characteristics of the hexose transporters mainly transcribed, due to the mode to form ATP. For this reason, we decided to measure the exponential growth of the deleted strains snf $1 \triangle$ and $h x k 2 \Delta$ under two concentrations of glucose: $0.005 \%(0.277 \mathrm{mM})$ and $5 \%(277 \mathrm{mM})$ that induce mitochondrial respiration and fermentation, respectively [5]. In this regard, a decrease in the specific growth rate is shown at $0.005 \%$ glucose (respiratory) in comparison to the growth at $5 \%$ glucose (fermentative) in the BY4741 strain (Fig. 1a), which corroborates the difference in the growth between a fermentative and respiratory glucose concentration. At $0.005 \%$ glucose, an increase in the specific growth rate occurs with the deletion of the genes SNF1 and HXK2 (Fig. 1b). However, only the strain $h x k 2 \Delta$ decreases the specific growth rate at $5 \%$ glucose (Fig. 1c). These results highlight the importance of $S N F 1$ in the growth at low-glucose concentrations that perform respiratory growth. Interestingly, HXK2 gene deletion has a dual effect on growth dependently of the glucose concentration.

\section{Influence of the overexpression of gene $H X K 2$ on the growth of $S$. cerevisiae}

Hxk2p is a key modulator of the glucose repression, however, is not clear when acts as a genetic modulator and when acts as a catalytic molecule. It has been reported that in low-glucosecontaining media $H X K 2$ expression is repressed by the transcriptional factors Rgt1p and Med8p $[25,26]$. At low-glucose concentrations probably the amount of Hxk2p regulates its function. To prove this idea, we made two strains that overexpress the HXK2 gene in the strains BY4741 and $h x k 2 \triangle$. The gene expression was under the GALl inducible promoter, which induces expression with galactose and represses expression with glucose. Besides, as a plasmid control, we used the plasmid pYES2.1 with the expression of lacZ gene to discard any effect produced for the extra DNA molecules within cells. Glucose supplementation of the pre-inoculum did not influence any changes in the growth of the recombinant strains BY4741//GAL1-HXK2 and hxk2 $/ / G A L 1-H X K 2$ in comparison with the parental strains BY4741 and $h x k 2 \Delta$ at $0.005 \%$ and 5\% glucose (Fig. 2ab). Interestingly, when galactose was used as a carbon source in the pre-inoculum to induce $H X K 2$ expression, the phenotype showed by the $h x k 2 \triangle$ strain at $0.005 \%$ in the recombinant strain $h x k 2 \triangle / / G A L 1-H X K 2$ was reverted, decreasing its growth compared to the BY4741 strain (Fig. 2c). At $5 \%$ glucose, $H X K 2$ expression also reverts the diminution on the growth of the $h x k 2 \Delta$ strain (Fig. 2d). The reverting phenotype of the strain $h x k 2 \triangle$ observed by $H X K 2$ overexpression also corroborates that the effects on growth detected in this strain are specific of the $H X K 2$ deletion. Nonetheless, $H X K 2$ overexpression in the BY4741 strain not display any change in the growth neither at $0.005 \%$ or $5 \%$ glucose (Fig. 2c-d). Overall, these data indicate that overexpression of the gene HXK2 did not have any effect on the growth of the BY4741.

340

341

342

343

344

345

346

347

348

349

350

351

352

353

354

355

\section{Impact of $H X K 2$ gene overexpression on S. cerevisiae mitochondrial respiration}

Another important phenotype exerted by glucose repression is the diminution of mitochondrial respiration, which is a well-known phenotype performed by the increase of the glucose concentration in S. cerevisiae (Fig. 3a). Therefore, the measuring of the mitochondrial respiration is a valuable indicator of glucose repression that could help to obtain further comprehension into the role of the SNF1 and $H X K 2$ genes in the glucose repression. The yeast cultures utilized to mitochondrial respiration assay came from a pre-inoculum supplemented with galactose to ensure the $H X K 2$ expression.

Interestingly, under the low concentration glucose $(0.005 \%)$ the $s n f 1 \triangle$ strain increased the basal and maximal mitochondrial respiration in contrast with the BY4741 strain (Fig. 3b-c). On the contrary, the strains $h x k 2 \triangle$ and $h x k 2 \triangle / / G A L 1-H X K 2$ did not show differences in basal and maximal mitochondrial respiration (Fig. 3b-c). At 5\% glucose the strains $h x k 2 \Delta$ and $h x k 2 \triangle / / G A L 1-H X K 2$ increased the basal respiration compared to the BY4741 strain (Fig. 3d); while only the strain $h x k 2 \triangle$ increased the maximal respiration (Fig. 3e). Importantly, between 
strains $h x k 2 \triangle$ and $h x k 2 \triangle / / G A L 1-H X K 2$ exists a difference in basal respiration with a diminution of oxygen consumption by $h x k 2 \triangle / / G A L 1-H X K 2$ strain (Fig. 3f). These data suggest that an increase of mitochondrial respiration may have a relation with the heightened in the growth in $s n f 1 \triangle$ strain at $0.005 \%$ glucose. Besides, the $h x k 2 \Delta$ complementation with $H X K 2$ gene does not have any effect in mitochondrial respiration at $0.005 \%$ glucose, whereas at $5 \%$ glucose a decrease on mitochondrial respiration is appreciated due to the $H X K 2$ expression. Probably the quantity of HXK2 expression is necessary to repress mitochondrial respiration in high-glucose concentrations.

\section{Influence of the deletion of the genes $H X K 2$, and $S N F 1$ on the transcription of $S$. cerevisiae BY4741}

To further understand whether the SNF1/HXK2 pathway modulates the growth and mitochondrial respiration through hexose transporters genetic regulation, we decide to measure the transcription of the hexoses transporters that allow us to recognize the interaction among these phenotypes and the pathway $S N F 1 / H X K 2$. Yeast cultures of these experiments came from a pre-inoculum grown with galactose to induce the expression in the recombinant strain $h x k 2 \triangle / / G A L 1-H X K 2$ and were grown in SC minimal media.

According to its affinity, we choose to quantify the transcription of the low-affinity hexose transporters genes (HXT1 and HXT3) and the high-affinity hexose transporters genes (HXT2 and HXT4). In the first place, the transcription in the strain BY4741 was used to corroborate the change in the transcription of $H X T 1-4$ genes in relation to the glucose concentration. HXT1 and HXT3 transcription augment at $5 \%$ of glucose in comparison with the yeast grown with $0.005 \%$ glucose (Fig 4a-b). On the contrary, transcription of the gene HXT2 decreases at $5 \%$ glucose with respect to the cultures grown at $0.005 \%$ glucose (Fig 4c). Although, HXT4 has been classified as a transporter of moderate or high affinity did not show any change in its transcription levels (Fig 4d). These data corroborate by an absolute quantification that HXT1 and HXT3 transcription is accord to its classification as low-affinity hexose transporters, likewise $H X T 2$ gene transcript induction, that occurs under low glucose concentration, corresponding to a high-affinity hexose transporter. However, HXT4 transcription did not change between the glucose concentrations evaluated.

Low-affinity hexose transporters like HXT1 and HXT3 are expressed at high-glucose concentrations mainly. At high glucose concentrations, Snflp is mainly inactive; snf $1 \triangle$ strain could resemble an inactive phenotype of this protein. Deletion of the gene SNF1 increases HXT1 transcription at 5\% glucose and does not have any effect in its transcription at $0.005 \%$ glucose (Fig. 5a-b); meanwhile, HXT3 transcription increased in the snf $1 \triangle$ strain at $0.005 \%$ and did not display differences in transcription at 5\% glucose (Fig. 5c-d). In the case of the HXK2 gene deletion and its complementing strain $h x k 2 \triangle / / G A L 1-H X K 2$ both showed a decrease in the transcription levels of the genes $H X T 1$ and HXT3 at 5\% glucose and at $0.005 \%$ glucose only HXT1 transcription showed a decrease (Fig. 5). Under low concentrations of glucose $(>0.1 \%)$ high-affinity transporters HXT2 and HXT4 are maximally expressed. Transcription of the HXT4 gene increased in the snf $1 \triangle$ strain at $0.005 \%$ and $5 \%$ glucose, whereas $H X T 2$ transcription was unaffected by $\operatorname{snf} 1 \triangle$ strain (Fig. 6). HXK2 deletion enhanced transcription of the genes $H X T 2$ and HXT4 only at 5\% glucose (Fig. 6). Importantly, overexpression of the gene $H X K 2$ reverted the increase in transcriptional levels of the genes HXT2 and HXT4 showed by the $h x k 2 \triangle$ strain at 5\% glucose (Fig. 6). In sum, these results suggest a negatively transcriptional regulation of $H X K 2$ upon high-affinity transporters $H X T 2$ and $H X T 4$, since overexpression of $H X K 2$ maintains the transcription at wild-type levels. However, transcription of low-affinity transporters has 406 repressed, even in the $h x k 2 \triangle / / G A L 1-H X K 2$ strain. Nonetheless, SNF1 deletion did not showed a 
407

408

409

410

411

412

413

414

415

416

417

418

419

420

421

422

423

424

425

426

427

428

429

430

431

432

433

434

435

436

437

438

439

440

441

442

443

444

445

446

447

448

449

450

451

452

453

454

455

456

pattern in transcription regulation, increasing $H X T 1$ and $H X T 4$ transcription at 5\% glucose and HXT2 and HXT4 at $0.005 \%$ glucose.

\section{The relationship among exponential growth, mitochondrial respiration, and hexose transporters transcription}

A correlational analysis was carried out to discriminate whether exists an effect of hexose transporters upon exponential growth and mitochondrial respiration in $S$. cerevisiae. The biochemical parameter Vmax was chosen to compare the relation of the hexose transporters with exponential growth and mitochondrial respiration, due to represents the velocity of glucose transport, which may impact the glycolytic flux. Vmax values were obtained from Reifenberger, Boles and Ciriacy [6]. Hexose transporters mainly transcribed, exponential growth values, and mitochondrial respiration were summarized in table 2 for $0.005 \%$ glucose, and table 3 for $5 \%$ glucose. For the correlational analysis, we only plotted the Vmax values of the hexose transporter genes with high transcriptional levels, in the case that were two genes with an increase of the transcriptional levels the average of them was plotted. We found a positive correlation between exponential growth and $\operatorname{Vmax}(\mathrm{r}=0.8452 ; P=0.0082 ; \mathbf{F i g} .7 \mathbf{a})$; indicating those hexose transporters with the major Vmax have the greater exponential growth, corresponding to the yeast cultures grown at $5 \%$ glucose, except for $h x k 2 \Delta$. The mitochondrial respiration showed a negative correlation with the Vmax values $(\mathrm{r}=-0.8201 ; P=0.0239$; Fig. $7 \mathbf{b})$; this data suggest that hexose transporters with low-values of Vmax promote mitochondrial respiration and hexose transporters with high-values of Vmax decrease mitochondrial respiration. Entirely, these data suggest a strong relation between glucose transporters with the exponential growth and mitochondrial respiration.

\section{Effect of the deletion of the genes MIG1 and SNF1 on the transcription of S. cerevisiae BY4741}

To a better understanding of the relation between the $S N F 1$ pathway and transcriptional levels of hexose transporters, we decided to make an additional experiment using a different $S$. cerevisiae strain BY4742 and its mutant in the SNF1 gene, to discard strain-specific effects. Additionally, we monitored the transcription of two additional hexose transporters HXT6 and HXT7, which are classified as high-affinity transporters. Besides, we use $0.01 \%(0.55 \mathrm{mM})$ as a low glucose concentration, to take a wider range of glucose concentrations. Finally, these experiments were carried out in YPD medium and came from a pre-inoculum grown with glucose.

To corroborate the expression of the hexose transporters according to its classification, we decided to measure its transcription under low glucose concentration $(0.01 \%)$ and high glucose concentration (5\%). Low-affinity transporters (HXT1 and HXT3) increased its transcription at 5\% glucose in comparison with transcription at $0.01 \%$ glucose (Fig. 8a-b). In the case of HXT2 and HXT4 transporters are considered as moderate-affinity or high-affinity transporters, we found that HXT2 augmented its transcription under low glucose concentration $(0.01 \%)$ as high-affinity transporters do, while HXT4 enhanced its transcription at 5\% glucose like a low-affinity transporter (Fig. 8c-d). Finally, the high-affinity transporters $H X T 6$ and $H X T 7$ did not display any change in its transcription with the two concentrations of glucose assayed (Fig. 8e-f). The profile of hexose transporter transcription is the same of the presented with BY4741 strain. However, the HXT4 presents a low-affinity transcription instead of a high-affinity pattern of transcription.

SNF1 deletion diminished the transcription of the HXT1 gene at $0.01 \%$ and did not change its transcription at 5\% glucose (Fig. 9a). HXT2 transcription was down at $0.01 \%$ and $5 \%$ glucose in the snfl $\triangle$ strain (Fig. 9c). Transcription of the HXT6 gene was decreased in snfl $\triangle$ strain grown at 5\% glucose with respect to BY4742 strain (Fig. 9e). A dual effect was observed in the transcription of the gene $H X T 7$, at $0.01 \%$ SNF1 deletion increased 10 times its transcription, 

HXT3 and HXT4 passed unaltered by the SNF1 gene deletion (Fig. 9b-d).

To strength the analysis of correlation between exponential growth and Vmax, we included the data obtained with the BY4742 strain and its deleting strain in SNF1. Exponential growth data and hexose transcription were summarized in table 4 for $0.01 \%$ glucose and table 5 for $5 \%$ glucose. The positive correlation between exponential growth and Vmax was maintained $(\mathrm{r}=$ 0.8495; $P=0.0005$; Fig. 10); showing a relation between the exponential growth at 5\% glucose concentration with the higher values of Vmax, and exponential growth at $0.005 \%$ and $0.01 \%$ glucose with the lower values of Vmax. This result suggests that Vmax of the hexose transporters could regulate the exponential growth of $S$. cerevisiae.

To gain knowledge about the hexose transporters and its regulation by the Snflp pathway, we decided to make a multivariate data analysis that included the transcriptional level data form hexose transporters obtained from strains BY4741 and BY4742 and its deleting strains in SNF1 gene. Importantly, PCA analysis showed that more dispersed clustering of hexose transcription corresponds to the moderate-affinity transporters especially the $H X T 2$ transcription (Fig. 11). HXT2 transcription was enhanced by low-glucose concentrations in both strains measured. At 5\% glucose $H X K 2$ deletion increased $H X T 2$ transcription, while $S N F 1$ deletion decreased it.

To corroborate the effect of the Snf1p/Hxk2p pathway upon HXT2 transcription was used MIG1 as an additional gene involved in the pathway. As expected, the deletion of the MIGl gene had a dual effect upon HXT2 transcription, increasing its transcription at 5\% glucose and decreasing it at $0.01 \%$ glucose (Fig. 12). These data indicate that the SNF1/HXK2/MIG1 pathway might have a specific role in the regulation of the $H X T 2$ gene transcription.

\section{Deletion of the genes RAG5 and SNF1 upon the growth of $K$. marxianus}

The regulation of the pathway $S N F 1 / H X K 2 / M I G 1$ upon hexose transport transcription, exponential growth, and mitochondrial respiration suggests that this pathway could regulate the switch between mitochondrial respiration and fermentation; this arises the question: whether the pathway SNF1/HXK2/MIG1 has a different role between Crabtree positives and Crabtree negative yeasts? To answer the last question, we used $K$. marxianus, which is a Crabtree negative yeast, that does not have the complex I of the electron transport chain, like $S$. cerevisiae. The orthologous genes of SNF1 and HXK2 of S. cerevisiae in K. marxianus are SNF1 and RAG5, respectively. At $0.005 \%$ glucose deletion of the $S N F 1$ gene decreased the growth of $S$. cerevisiae, while in $K$. marxianus SNF1 deletion increased the growth (Fig. 13a). In the case of the HXK2 deletion did not have any effect on the growth of $S$. cerevisiae at $0.005 \%$ glucose, meanwhile, $R A G 5$ deletion augmented the growth of K. marxianus (Fig. 13a). SNF1 gene deletion lessened the growth of $S$. cerevisiae at $5 \%$ glucose, likewise, Kmsnfl $\triangle$ showed a minor growth than the parental strain of $K$. marxianus at $5 \%$ glucose (Fig. 13b). At $5 \%$ glucose $s c h x k 2 \Delta$ and kmrag $5 \Delta$ strains displays a decrease in the growth of $S$. cerevisiae and $K$. marxianus, respectively (Fig. 13b). Overall, these data suggest the opposite role in the growth of the pathway SNF1/RAG5 in $K$. marxianus promoting the growth at low-glucose concentration and decreasing the growth in highglucose concentration. Additionally, at $0.005 \%$ is evident the divergent role on the growth of the pathway $S N F 1 / H X K 2-R A G 5$ between $S$. cerevisiae and K. marxianus.

501

502

503

504

505

506

507

\section{Impact of the via $S N F 1 / R A G 5$ pathway on the mitochondrial respiration of $K$. marxianus}

To better understand how the pathway SNF1/RAG5 impacts in the glucose-sensing of $K$. marxianus, that not presents the Crabtree effect, we measure its mitochondrial respiration. Interestingly, $K$. marxianus displays a diminution of mitochondrial respiration with the augment of the glucose concentration such as occur in S. cerevisiae (Fig. 14a). However, the difference in 
508

509

510

511

512

513

514

515

516

517

518

519

520

521

522

523

524

525

526

527

528

529

530

531

532

533

534

535

536

537

538

539

540

541

542

543

544

545

546

547

548

549

550

551

552

553

554

555

556

557

558 basal mitochondrial respiration between $K$. marxianus and $S$. cerevisiae are 14.5 times higher at $0.005 \%$ glucose and 105 times higher at $5 \%$ glucose (Fig. 14a). This result suggests that $K$. marxianus, although it is not a Crabtree positive yeast, responds similarly to glucose, lessening its mitochondrial respiration with lower levels of glucose.

The deletion of the SNF1 gene has the same influence on mitochondrial respiration, decreasing it with respect to its parental strains at $0.005 \%$ glucose (Fig. 14b-c). In the case of the HXK2/RAG5 deletion, there was an increment in mitochondrial respiration in S. cerevisiae and diminishing in $K$. marxianus when both were grown at $0.005 \%$ glucose (Fig 14b-c). At $5 \%$ glucose, the deletion of the SNF1/HXK2-RAG5 genes has contrasting effects: increasing mitochondrial respiration in $S$. cerevisiae and decreasing mitochondrial respiration in $K$. marxianus (Fig 14d-e). These results highlight the opposite role of the SNF1/HXK2-RAG5 pathway under high-glucose concentration that exerts the Crabtree effect, suggesting its participation in the molecular mechanism. Even, at $0.005 \%$ glucose $H X K 2-R A G 5$ deletion also had a differential influence upon mitochondrial respiration.

\section{Discussion}

The Crabtree effect mediated by glucose repression is a metabolic phenomenon with biomedical and biotechnological importance [27]. For example, in S. cerevisiae the ethanol production also occurs during the Crabtree effect under oxygen presence $[28,29]$. The Warburg effect metabolic analogous to the Crabtree effect is present in the majority of the cancerogenic cells and its part of the cancer etiology $[2,30]$. Nonetheless, the key piece to explain the molecular mechanism of the Crabtree effect is maintained elusive. Glucose transport could play an important role in the modulation of the glycolytic flux since it increasing is essential to induce the Crabtree effect. The wide-range of biochemical characteristics of the hexose transporters of S. cerevisiae implies a highly adapted metabolism to the uptake of sugars in different circumstances. However, the metabolic control associating hexose transporters expression with the Crabtree phenotype is not totally explained yet. The pathway Snf1p/Hxk2p/Mig1p has been associated with the transcriptional regulation of the hexose transporters $[12,16-18,31]$. Besides, the pathway Snf1p/Hxk2p/Mig1p has also been identified in the modulation of some phenotypes associated with the Crabtree effect, such as mitochondrial respiration [10], glycolytic flux [10], and fermentation [12]. Nevertheless, it has not been directly identified the direct genetic regulation of the hexose transporters with modulation of the Crabtree effect phenotypes by Snf1p/Hxk2p/Mig1p pathway. For this reason, the aim of this study was to identify the association between the transcriptional levels of hexose transporters with the growth and mitochondrial respiration, modulated by the Snf1p/Hxk2p/Mig1p pathway. In view of this, we provide evidence that the deletion of the $S N F 1$ and $H X K 2$ disturbs the exponential growth of $S$. cerevisiae in a glucose-dependent manner. Besides, snfl $\triangle$ and $h x k 2 \Delta$ strains impact in the mitochondrial respiration of $S$. cerevisiae in a glucose-dependent manner. The transcriptional levels of hexose transporters were also affected by the deletion of the SNF1 and HXK2 with a differential pattern in response to glucose concentration. A positive correlation was found between exponential growth and Vmax of the hexose transporters mainly transcribed; while a negative correlation was detected between mitochondrial respiration and Vmax of the hexose transporters mainly transcribed. Transcription of the gene $H X T 2$ was the most affected by the deletion of the pathway $S N F 1 / H X K 2 / M I G 1$ in the glucose concentrations tested $(0.005 \%, 0.01 \%$, and 5\%). Finally, the deletion of the orthologous genes SNF1 and $H X K 2$ in the Crabtree negative yeast, $K$. marxianus, has a differential effect in exponential growth and mitochondrial respiration in comparison with $S$. cerevisiae (Crabtree positive). In general, these results indicate that the SNF1/HXK2/MIG1 pathway regulates the transcriptional levels of hexose transporters and this modulates the exponential growth and mitochondrial respiration. 
Mitochondrial respiration and fermentation are pathways that enable microorganism division by ATP formation. It is expected that the alteration of the exponential growth rate, allow screening changes in bioenergetic metabolism [5]. Intriguingly, the increase in growth is a phenotype well documented during the Crabtree effect and with its analogous the Warburg effect, this higher growth occurs mainly under fermentation, which produces 2 mol of ATP per mol of glucose, 9 times lower than mitochondrial respiration in $S$. cerevisiae and 15-18 times lesser than mammalian cells. To maintain this growth and its concomitant ATP demands, the cells should be uptaken higher amounts of glucose through its hexose transporters. The glucose repression regulates those changes in the metabolism through the Hxk2p and Mig1p, repressing the transcription of genes that participates in the respiratory energetic metabolism [1]. In the case of Snf1p is a kinase that inhibits Hxk2p and Mig1p by phosphorylation, playing a role as a negative regulator of glucose repression. In the first part of this study, we evaluate the exponential growth, with the basis that changes in the energetic metabolism should impact in this type of growth. SNF1 and HXK2 gene deletions modified the exponential growth of $S$. cerevisiae with important differences. The $S N F 1$ gene deletion increases the exponential growth at $0.005 \%$ glucose (Fig. 1b) when it came from an inoculum grown with $\mathrm{SC}$ medium, whereas the same gene deletion decreased the exponential growth at $0.005 \%$ glucose when it came from an inoculum grown with the YPD medium (Fig 13a). A similar phenotype was observed with $H X K 2$ gene deletion, which increasing exponential growth when cells were precultivated in SC medium (Fig. 1b) and no change the exponential growth when the pre-inoculum was made with YPD medium (Fig. 13a). These data highlight the dependence of the exponential growth exerted by SNF1 and HXK2 genes deletion upon nutrient availability. The response of mitochondrial respiration to both SNF1 and $H X K 2$ genes deletion was similar to the phenotype observed with exponential growth. At $0.005 \%$ glucose $\operatorname{snf} 1 \triangle$ strain augments basal respiration when cells were precultivated in SC medium (Fig 3b) and the basal respiration was lessen in cultures coming from a pre-inoculum grown in YPD medium (Fig. 14b). The $h x k 2 \Delta$ strain did not change the basal respiration at $0.005 \%$ glucose (Fig 3b) when it came from a pre-inoculum grown with SC medium, while increases the basal respiration at $0.005 \%$ glucose when cells were precultivated with YPD medium (Fig. 14b). These results remark the association of the exponential growth with mitochondrial respiration and also underline the intricate involvement of SNF1/HXK2 with the nutrient disposition in the media, highlighting the importance of describing the pre-inoculum to the better comparison of data.

As expected, the $H X K 2$ gene deletion reverts two important phenotypes observed during the Crabtree, diminishing the exponential growth (Fig. 2d and 13b) and increasing the mitochondrial respiration both at 5\% glucose (Fig. 3d and 14d). A diminution in the exponential growth through the doubling time has also been reported, in the $h x k 2 \Delta$ strain grown in YPD medium and SD minimal medium coming from S. cerevisiae DBY2230 genetic background [32]. Since SNF1 has opposite participation in the glucose repression to $H X K 2$, we expected to observe the phenotypes related to the Crabtree effect unchanged. The $\operatorname{snfl} \triangle$ strain precultivated in the SC medium did not change either exponential growth (Table 3) and mitochondrial respiration (Fig. 3d). However, snfl $\triangle$ strain pre-inoculated in the YPD medium showed a slight decrease in exponential growth (Fig. 13b) and an increase in mitochondrial respiration at 5\% glucose (Fig. 14d). The increase in the mitochondrial respiration was also observed in $\operatorname{snf} 1 \triangle$ cells grown in SC medium with $2 \%$ glucose [12] and in snfl $\triangle$ cells grown in YPD medium supplemented with $10 \%$ glucose [10]. These phenotypes make clear the participation of the Snflp at high-glucose concentrations, since it impairs the mitochondrial respiration. Interestingly, Snflp regulates the arresting $\square$ related trafficking adaptor Rod $1 \mathrm{p} /$ Art $4 \mathrm{p}$, which mediates the endocytosis of the high $\square$ affinity glucose transporter Hxt6p at high glucose concentrations [31]. Besides, the SNF1 gene lacking stimulates Hxt1p and Hxt3p endocytosis and degradation in the vacuole [33]. Importantly, overexpression of low-affinity transporters Hxt1p and Hxt3p rescues snfl $\triangle$ growth supplemented with $0.01 \%$ 2-deoxyglucose [33]. These studies remark the strength relation 
between $S N F 1$ and the hexose transporters expression. Additionally, these results indicate the

611

612

613

614

615

616

617

618

619

620

621

622

623

624

625

626

627

628

629

630

631

632

633

634

635

636

637

638

639

640

641

642

643

644

645

646

647 participation of the $S N F 1 / H X K 2$ in the exponential growth and the mitochondrial respiration, two important phenotypes observed in the Crabtree effect.

$S$. cerevisiae count with a numerous family of hexose transporters that have a wide range of biochemical characteristics and is according to its affinity $(\mathrm{km})$ that hexose transporters were classified [34]. However, little attention has been putting in the Vmax, which describes the capacity of each hexose transporter to uptake sugar in a unit of time. Remarkably, a positive correlation has been found between the sugar uptake rate and ethanol production rate in $S$. cerevisiae [7,8], which suggests that the key piece in the establishment of the Crabtree effect might be the glucose transport. Here, we found that exponential growth (Fig. 7a and Fig. 10) and mitochondrial respiration (Fig. 7b) correlates with the Vmax of the mainly transcribed hexose transporters. These results reveal that rate of glucose uptake (Vmax) could be an important mediator in the establishment of the phenotypes (augmented growth and mitochondrial respiration repressed) related to the Crabtree effect. In support of this idea, a linear correlation between the natural logarithms of the Vmax of hexose transporters and the glucose consumption rate in $S$. cerevisiae was found [7]. In addition, when $S$. cerevisiae cultures grown in aerobic chemostat using ethanol as a carbon source were exposed to glucose (fructose) pulse, the strain that only expressed Hxt1p transporter changed its phenotype to a respiro-fermentative metabolism as did the wild-type strain, whereas strain expressing Hxt7p transporter maintained respiratory metabolism [35]. Importantly, the PCA revealed that $H X T 2$ transcription was the most affected by the SNF1/HXK2/MIG1 (Fig. 11), which might have important effects on the phenotypes exerted by this pathway. Overall, these results highlight the relationship between hexose transporters activity, glucose uptake rate, and mitochondrial respiration/fermentation. Additionally, our results reveal that the SNF1/HXK2/MIG1 pathway regulates in a transcriptional way the hexose transporters, this pathway also showed a linear correlation with the exponential growth and mitochondrial respiration, two characteristic phenotypes presented during the Crabtree effect.

\section{Funding and additional information}

This work was supported by the grant: PROMEP program (ITESCH-EXB-002) awarded to LAMP that includes the scholarship grant to JGMG.

\section{Conflict of interest statement}

The authors declare that they have no conflicts of interest with the contents of this article.

\section{References}

[1] O. Kayikci, J. Nielsen, Glucose repression in Saccharomyces cerevisiae, FEMS yeast research, 15 (2015) fov068.

648 [2] R. Diaz-Ruiz, M. Rigoulet, A. Devin, The Warburg and Crabtree effects: On the origin of cancer cell energy metabolism and of yeast glucose repression, Biochimica et biophysica acta, 1807 (2011) 568-576.

651 [3] R. Diaz-Ruiz, N. Averet, D. Araiza, B. Pinson, S. Uribe-Carvajal, A. Devin, M. Rigoulet,

652

653

654

655

656 Mitochondrial oxidative phosphorylation is regulated by fructose 1,6-bisphosphate. A possible role in Crabtree effect induction?, The Journal of biological chemistry, 283 (2008) 26948-26955.

[4] S. Rodríguez-Enríquez, O. Juárez, J.S. Rodríguez-Zavala, R. Moreno-Sánchez, Multisite control of the Crabtree effect in ascites hepatoma cells, European Journal of Biochemistry, 268 (2001) 2512-2519.

657 [5] I.K. Olivares-Marin, L.A. Madrigal-Perez, M. Canizal-Garcia, B.E. Garcia-Almendarez, J.C.

658 Gonzalez-Hernandez, C. Regalado-Gonzalez, Interactions between carbon and nitrogen sources

659 depend on RIM15 and determine fermentative or respiratory growth in Saccharomyces cerevisiae,

660 Appl Microbiol Biotechnol, 102 (2018) 4535-4548. 
661 [6] E. Reifenberger, E. Boles, M. Ciriacy, Kinetic characterization of individual hexose

662

663

664

665

666

667

668 transporters of Saccharomyces cerevisiae and their relation to the triggering mechanisms of glucose repression, European Journal of Biochemistry, 245 (1997) 324-333.

[7] K. Elbing, C. Larsson, R.M. Bill, E. Albers, J.L. Snoep, E. Boles, S. Hohmann, L. Gustafsson, Role of hexose transport in control of glycolytic flux in Saccharomyces cerevisiae, Applied and environmental microbiology, 70 (2004) 5323-5330.

669

670

[8] D.H. Huberts, B. Niebel, M. Heinemann, A flux-sensing mechanism could regulate the switch between respiration and fermentation, FEMS yeast research, 12 (2012) 118-128.

671 [9] D.G. Hardie, D. Carling, M. Carlson, The AMP-Activated/SNF1 Protein Kinase Subfamily: Metabolic Sensors of the Eukaryotic cell?, Annual review of biochemistry, 67 (1998) 821-855.

672 [10] C. Martinez-Ortiz, A. Carrillo-Garmendia, B.F. Correa-Romero, M. Canizal-Garcia, J.C. Gonzalez-Hernandez, C. Regalado-Gonzalez, I.K. Olivares-Marin, L.A. Madrigal-Perez, SNF1 controls the glycolytic flux and mitochondrial respiration, Yeast, 36 (2019) 487-494.

674

675 [11] L.A. Madrigal-Perez, G.M. Nava, J.C. Gonzalez-Hernandez, M. Ramos-Gomez, Resveratrol increases glycolytic flux in Saccharomyces cerevisiae via a SNF1-dependet mechanism, Journal of bioenergetics and biomembranes, 47 (2015) 331-336.

678 [12] R. Nicastro, F. Tripodi, C. Guzzi, V. Reghellin, S. Khoomrung, C. Capusoni, C. Compagno, C. Airoldi, J. Nielsen, L. Alberghina, P. Coccetti, Enhanced amino acid utilization sustains growth of cells lacking Snf1/AMPK, Biochimica et biophysica acta, 1853 (2015) 1615-1625.

680

681 [13] P. Fernandez-Garcia, R. Pelaez, P. Herrero, F. Moreno, Phosphorylation of yeast hexokinase 2 regulates its nucleocytoplasmic shuttling, The Journal of biological chemistry, 287 (2012)

683

684 42151-42164.

685

686

687

688

689

690

691

692

693

694

695

696

697

698

699

700

701

702

703

704

705

[14] M. Carlson, Glucose repression in yeast, Current Opinion in Microbiology, 2 (1999) 202207.

[15] M. Vega, A. Riera, A. Fernandez-Cid, P. Herrero, F. Moreno, Hexokinase 2 Is an Intracellular Glucose Sensor of Yeast Cells That Maintains the Structure and Activity of Mig1 Protein Repressor Complex, The Journal of biological chemistry, 291 (2016) 7267-7285.

[16] S. Ozcan, M. Johnston, Two different repressors collaborate to restrict expression of the yeast glucose transporter genes HXT2 and HXT4 to low levels of glucose., Molecular and Cellular Biology, 16 (1996) 5536-5545.

[17] S.L. Westergaard, A.P. Oliveira, C. Bro, L. Olsson, J. Nielsen, A systems biology approach to study glucose repression in the yeast Saccharomyces cerevisiae, Biotechnology and bioengineering, 96 (2007) 134-145.

[18] J.O. Westholm, N. Nordberg, E. Muren, A. Ameur, J. Komorowski, H. Ronne, Combinatorial control of gene expression by the three yeast repressors Mig1, Mig2 and Mig3, BMC genomics, 9 (2008) 601.

[19] A. Baudin, O. Ozier-Kalogeropoulos, A. Denouel, F. Lacroute, C. Cullin, A simple and efficient method for direct gene deletion in Saccharomyces cerevisiae, Nucleic acids research, 21 (1993) 3329-3330.

[20] A. Wach, A. Brachat, R. Pöhlmann, P. Philippsen, New heterologous modules for classical or PCR-based gene disruptions in Saccharomyces cerevisiae, Yeast, 10 (1994) 1793-1808.

[21] D. Gietz, A. St Jean, R.A. Woods, R.H. Schiestl, Improved method for high efficiency transformation of intact yeast cells, Nucleic acids research, 20 (1992) 1425.

[22] R.D. Gietzt, R.H. Schiestls, A.R. Willems, R.A. Woods, Studies on the transformation of intact yeast cells by the LiAc/SS-DNA/PEG procedure, Yeast, 11 (1995) 355-360.

[23] M.F. Tello-Padilla, A.Y. Perez-Gonzalez, M. Canizal-Garcia, J.C. Gonzalez-Hernandez, C. Cortes-Rojo, I.K. Olivares-Marin, L.A. Madrigal-Perez, Glutathione levels influence chronological life span of Saccharomyces cerevisiae in a glucose-dependent manner, Yeast, 35 (2018) 387-396.

710 [24] T. Metsalu, J. Vilo, ClustVis: a web tool for visualizing clustering of multivariate data using 711 Principal Component Analysis and heatmap, Nucleic acids research, 43 (2015) W566-570. 

for transcriptional repression of the HXK2 gene in Saccharomyces cerevisiae, The Biochemical journal, 388 (2005) 697-703.

[26] A. Palomino, P. Herrero, F. Moreno, Tpk3 and Snf1 protein kinases regulate Rgt1 association with Saccharomyces cerevisiae HXK2 promoter, Nucleic acids research, 34 (2006) 1427-1438.

[27] N. Hammad, M. Rosas-Lemus, S. Uribe-Carvajal, M. Rigoulet, A. Devin, The Crabtree and Warburg effects: Do metabolite-induced regulations participate in their induction?, Biochimica et biophysica acta, 1857 (2016) 1139-1146.

[28] Verduyn C., Zomerdijk T.P.L., Van Dijken J.P., S. W.A., Continuous measurement of ethanol production by aerobic yeast suspension with an enzyme electrode, Appl Microbiol Biotechnol 19 (1984) 181-185.

[29] K. Otterstedt, C. Larsson, R.M. Bill, A. Stahlberg, E. Boles, S. Hohmann, L. Gustafsson, Switching the mode of metabolism in the yeast Saccharomyces cerevisiae, EMBO reports, 5 (2004) 532-537.

[30] E. de Alteriis, F. Carteni, P. Parascandola, J. Serpa, S. Mazzoleni, Revisiting the Crabtree/Warburg effect in a dynamic perspective: a fitness advantage against sugar-induced cell death, Cell Cycle, 17 (2018) 688-701.

[31] V. Llopis-Torregrosa, A. Ferri-Blazquez, A. Adam-Artigues, E. Deffontaines, G.P. van Heusden, L. Yenush, Regulation of the Yeast Hxt6 Hexose Transporter by the Rod1 alphaArrestin, the Snf1 Protein Kinase, and the Bmh2 14-3-3 Protein, The Journal of biological chemistry, 291 (2016) 14973-14985.

734 [32] H. Ma, L.M. Bloom, Z.M. Zhu, C.T. Walsh, D. Botstein, Isolation and characterization of mutations in the HXK2 gene of Saccharomyces cerevisiae., Molecular and Cellular Biology, 9 (1989) 5630-5642.

[33] A.F. O'Donnell, R.R. McCartney, D.G. Chandrashekarappa, B.B. Zhang, J. Thorner, M.C. Schmidt, 2-Deoxyglucose impairs Saccharomyces cerevisiae growth by stimulating Snf1regulated and alpha-arrestin-mediated trafficking of hexose transporters 1 and 3, Mol Cell Biol, 35 (2015) 939-955.

[34] J. Horak, Regulations of sugar transporters: insights from yeast, Current genetics, 59 (2013) $1-31$.

[35] D. Bosch, M. Johansson, C. Ferndahl, C.J. Franzen, C. Larsson, L. Gustafsson, Characterization of glucose transport mutants of Saccharomyces cerevisiae during a nutritional upshift reveals a correlation between metabolite levels and glycolytic flux, FEMS yeast research, 8 (2008) 10-25.

[36] J. Ye, G. Coulouris, I. Zaretskaya, I. Cutcutache, Ç, S. Rozen, T.L. Madden, Primer-BLAST: A tool to design target-specific primers for polymerase chain reaction, BMC Bioinformatics, 13 (2012) 134.

[37] M.A. Teste, M. Duquenne, J.M. Francois, J.L. Parrou, Validation of reference genes for quantitative expression analysis by real-time RT-PCR in Saccharomyces cerevisiae, BMC molecular biology, 10 (2009) 99.

\section{Figure legends}

Fig 1. Influence of the deletion of the genes $S N F 1$ and $H X K 2$ on the exponential growth of $S$. cerevisiae at different glucose concentrations. The exponential growth was determined with the specific growth rate, which was calculated fitting data with the exponential growth rate. a) Specific growth rate of BY4741 at $0.005 \%$ and $5 \%$ glucose; b) represents growth at $0.005 \%$ glucose; c) represents growth at $5 \%$ glucose. The data represents mean \pm standard deviation of four independent experiments with two technical replicates. For panels b) and c) means were

761 compared using one-way ANOVA followed by a Dunnett multiple comparison vs. BY4741 
$(* * * * P<0.0001)$. Two-tailed unpaired $t$-test was used to compare means of the panel a) $(* * * * P$ $<0.0001)$.

Fig 2. Effect of the $H X K 2$ gene overexpression on the exponential growth of S. cerevisiae strains BY4741 and $h x k 2 \Delta$. The specific growth rate was calculated fitting data with the exponential growth rate. a) Represents growth at $0.005 \%$ glucose coming from a pre-inoculum supplemented with glucose; b) represents growth at $5 \%$ glucose coming from a pre-inoculum supplemented with glucose; c) represents growth at $0.005 \%$ glucose coming from a pre-inoculum supplemented with galactose; d) represents growth at 5\% glucose coming from a pre-inoculum supplemented with galactose. The data represents mean \pm standard deviation of four independent experiments with two technical replicates. Means were compared using one-way ANOVA followed by a Dunnett multiple comparison vs. BY4741 $(* P<0.05 ; * * P<0.01 ; * * * P<0.001 ; * * * * P<0.0001)$.

Fig 3. Mitochondrial respiration of the strains BY4741, $h x k 2 \Delta$, snfl $\triangle$, and $h x k 2 \triangle / / G A L 1-H X K 2$ at $0.005 \%$ and $5 \%$ glucose. Basal and maximal respiration were obtained from cultures in which pre-inoculum was grown with galactose in an SC medium. a) Basal respiration of BY4741 strain at $0.005 \%$ and $5 \%$ glucose; b) basal respiration of the strains BY4741, hxk2 $\triangle$, snf $1 \triangle$, and $h x k 2 \triangle / / G A L 1-H X K 2$ at $0.005 \%$ glucose; c) maximal respiration of the strains BY4741, $h x k 2 \Delta$, $s n f 1 \triangle$, and $h x k 2 \triangle / G A L 1-H X K 2$ at $0.005 \%$ glucose; d) basal respiration of the strains BY4741, $h x k 2 \Delta$, snfl $\triangle$, and $h x k 2 \triangle / / G A L 1-H X K 2$ at $5 \%$ glucose; e) maximal respiration of the strains BY4741, $h x k 2 \Delta$, snfl $\triangle$, and $h x k 2 \triangle / / G A L 1-H X K 2$ at $5 \%$ glucose; f) comparison of the basal respiration between $h x k 2 \triangle$ and $h x k 2 \triangle / / G A L 1-H X K 2$ strains at $5 \%$ glucose. The data represents mean \pm standard deviation of three independent experiments. For panels b), c), d), and e) means were compared using one-way ANOVA followed by a Dunnett multiple comparison $v s$. BY4741 $(* * P<0.01 ; * * * P<0.001$;**** $P<0.0001$ ). For panels a) and f) means were compared with a two-tailed unpaired $t$-test $(* P<0.05 ; * * * P<0.001)$.

Fig 4. Transcription of the genes HXT1, HXT2, HXT3, and HXT4 in the BY4741 strain at $0.005 \%$ and $5 \%$ glucose. Absolute quantification was made using a UBC6 as a reference gene, from $S$. cerevisiae cultures in which pre-inoculum was grown with galactose in an SC medium. a) Transcription of HXT1 gene; b) transcription of HXT3 gene; c) Transcription of HXT2 gene; d) transcription of HXT4 gene. The data represents mean \pm standard deviation of three independent experiments with two technical replicates. Means were compared with a two-tailed unpaired $t$-test ( $* P<0.05 ; * * * P<0.001 ; * * * * P<0.0001 ;$ ns, non-significant).

Fig 5. Transcription of the low-affinity hexose transporters genes HXT1 and HXT3 in the BY4741, $h x k 2 \Delta$, snfl 1 , and $h x k 2 \triangle / / G A L 1-H X K 2$ strains at $0.005 \%$ and $5 \%$ glucose. Absolute quantification was made using a UBC6 as a reference gene, from $S$. cerevisiae cultures in which pre-inoculum was grown with galactose in an SC medium. a) Represents $H X T 1$ gene transcription at $0.005 \%$; b) represents $H X T 1$ gene transcription at 5\%; c) represents $H X T 3$ gene transcription at $0.005 \%$; b) represents $H X T 3$ gene transcription at $5 \%$. The data represents mean \pm standard deviation of three independent experiments. Means were compared using one-way ANOVA followed by a Dunnett multiple comparison $v$. BY4741 $\left({ }^{*} P<0.05 ; * * P<0.01\right.$; *** $P<0.001$; $* * * * P<0.0001)$.

Fig 6. Transcription of the high-affinity hexose transporters genes HXT2 and HXT4 in the BY4741, $h x k 2 \Delta$, snfl $1 \Delta$, and $h x k 2 \triangle / / G A L 1-H X K 2$ strains at $0.005 \%$ and $5 \%$ glucose. Absolute quantification was made using a $U B C 6$ as a reference gene, from $S$. cerevisiae cultures in which pre-inoculum was grown with galactose in an SC medium. a) Represents $H X T 2$ gene transcription at $0.005 \%$; b) represents $H X T 2$ gene transcription at 5\%; c) represents $H X T 4$ gene transcription at

$8120.005 \%$; b) represents $H X T 4$ gene transcription at $5 \%$. The data represents mean \pm standard 
deviation of three independent experiments. Means were compared using one-way ANOVA

814

815

816

817

818

819

820

821

822

823

824

825

826

827

828

829

830

831

832

833

834

835

836

837

838

839

840

841

842

843

844

845

846 followed by a Dunnett multiple comparison $v$. BY4741 $(* P<0.05 ; * * P<0.01$; *** $P<0.001)$.

Fig 7. Analysis of correlation between exponential growth/mitochondrial respiration with Vmax of hexose transporters. Pearson`s correlational analysis was carried out to evaluated the correlation between exponential growth/mitochondrial respiration with Vmax. a) Exponential growth vs. Vmax; b) mitochondrial respiration vs. Vmax. Pearson`s correlation was calculated with the statistical package GraphPad Prism 6.00 for Macintosh (GraphPad Software).

Fig 8. Transcription of the genes HXT1, HXT2, HXT3, HXT4, HXT6, and HXT7 in the BY4742 strain at $0.01 \%$ and $5 \%$ glucose. Absolute quantification was made using a UBC6 as a reference gene, from $S$. cerevisiae cultures in which pre-inoculum was grown with glucose in a YPD medium. a) Transcription of $H X T 1$ gene; b) transcription of $H X T 3$ gene; c) Transcription of $H X T 2$ gene; d) transcription of HXT4 gene; e) transcription of HXT6 gene; f) transcription of HXT7 gene. The data represents mean \pm standard deviation of three independent experiments with two technical replicates. Means were compared with a two-tailed unpaired $t$-test $(* P<0.05$; $* * P<$ $0.01 ; * * * P<0.001)$.

Fig 9. Transcription of the genes HXT1, HXT2, HXT3, HXT4, HXT6, and HXT7 in the BY4742 and $\operatorname{snf} 1 \triangle$ strains at $0.01 \%$ and $5 \%$ glucose. Absolute quantification was made using a UBC6 as a reference gene, from $S$. cerevisiae cultures in which pre-inoculum was grown with glucose in a YPD medium. a) Transcription of HXT1 gene; b) transcription of HXT3 gene; c) Transcription of HXT2 gene; d) transcription of HXT4 gene; e) transcription of HXT6 gene; f) transcription of HXT7 gene. The data represents mean \pm standard deviation of three independent experiments with two technical replicates. Means were compared with a two-tailed unpaired $t$-test $(* P<0.05 ; * * P$ $<0.01 ; * * * P<0.001)$.

Fig 10. Analysis of correlation between exponential growth with Vmax of hexose transporters. Pearson`s correlational analysis was carried out to evaluated the correlation between exponential growth with Vmax. Black dots represent $S$. cerevisiae cultures in which pre-inoculum was grown with galactose in an SC medium. Blue dots represent $S$. cerevisiae cultures in which pre-inoculum was grown with glucose in a YPD medium. Pearson`s correlation was calculated with the statistical package GraphPad Prism 6.00 for Macintosh (GraphPad Software).

Fig 11. PCA analysis of $H X T$ transcription in the strain BY4741 and BY4742, and its strains deleted in the $S N F 1$ gene. Original values are $\ln (\mathrm{x}+1)$-transformed. No scaling is applied to rows; probabilistic PCA is used to calculate principal components. $X$ and $Y$ axis show principal component 1 and principal component 2 that explains $67.5 \%$ and $32.5 \%$ of the total variance, respectively. Prediction ellipses are such that with probability 0.95 , a new observation from the same group will fall inside the ellipse. $\mathrm{N}=20$ data points.

852

853

854

855

856

857

858

859

860

861

Fig 12. Influence of the MIG1 deletion in the transcription of the HXT2 gene at $0.01 \%$ and $5 \%$ glucose. Absolute quantification was made using a $U B C 6$ as a reference gene, from $S$. cerevisiae cultures in which pre-inoculum was grown with glucose in a YPD medium. The data represents mean \pm standard deviation of three independent experiments with two technical replicates. Means were compared with a two-tailed unpaired $t$-test $(* P<0.05)$.

Fig 13. Influence of deletion of the genes $S N F 1$ and $H X K 2 / R A G 5$ in the growth of $S$. cerevisiae and $K$. marxianus. The exponential growth was represented trough the specific growth rate of the strain deleted in the genes $S N F 1$ and $H X K 2$. In the case of $S$. cerevisiae the strains were named as 862

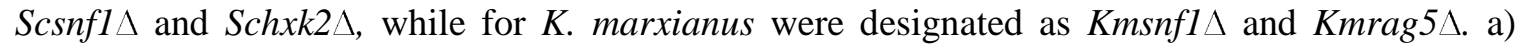


864

865

866

867

868

869

870

871

872

873

874

875

876

877

878

879

880

881

882

883

884

885

886

887

888

889

Represents growth at $0.005 \%$ glucose; b) represents growth at $5 \%$ glucose. The data represents mean \pm standard deviation of four independent experiments with two technical replicates. Means were compared using one-way ANOVA followed by a Dunnett multiple comparison vs. $S$. cerevisiae and $K$. marxianus $(* P<0.05$; ** $P<0.01$; *** $P<0.001)$.

Fig 14. Effect of deletion of the genes $S N F 1$ and $H X K 2 / R A G 5$ in the mitochondrial of $S$. cerevisiae and $K$. marxianus at $0.005 \%$ and $5 \%$ glucose. Basal and maximal respiration were obtained from cultures in which pre-inoculum was grown with glucose in a YPD medium. a) Basal respiration of $S$. cerevisiae and $K$. marxianus strains at $0.005 \%$ and $5 \%$ glucose; b) basal respiration of the strains $S$. cerevisiae and $K$. marxianus and its deletant strains in the genes $S N F 1$ and $H X K 2 / R A G 5$ at $0.005 \%$ glucose; c) maximal respiration of the strains $S$. cerevisiae and $K$. marxianus and its deletant strains in the genes SNF1 and HXK2/RAG5 at $0.005 \%$ glucose; d) basal respiration of the strains $S$. cerevisiae and $K$. marxianus and its deletant strains in the genes $S N F 1$ and $H X K 2 / R A G 5$ at 5\% glucose; e) maximal respiration of the strains $S$. cerevisiae and $K$. marxianus and its deletant strains in the genes SNF1 and HXK2/RAG5 at 5\% glucose. The data represents the mean \pm standard deviation of three independent experiments. For panels b), c), d), and e) means were compared using one-way ANOVA followed by a Dunnett multiple comparison vs. S. cerevisiae or K. marxianus $\left({ }^{*} P<0.05 ; * * P<0.01 ; * * * P<0.001\right)$. For panel a) means were compared with a two-tailed unpaired $t$-test $(* * * P<0.001 ; * * * P<0.001)$.

Table 1. RT- qPCR hexose transporters primers

\begin{tabular}{|c|c|c|c|c|}
\hline Gene & Direction & Primer sequence & $\begin{array}{c}\text { Fragm } \\
\text { ent } \\
\text { length }\end{array}$ & $\begin{array}{l}\text { Referenc } \\
\text { e }\end{array}$ \\
\hline$H X T 1$ & $\begin{array}{l}\text { Forward } \\
\text { Reverse }\end{array}$ & $\begin{array}{l}5^{\prime}-\text { GCCCACCTGACCATCCATAC -3' } \\
5^{\prime}-\text { AACCGACAGCCTGGAAAACA -3' }\end{array}$ & 222 bp & [36] \\
\hline$H X T 2$ & $\begin{array}{l}\text { Forward } \\
\text { Reverse }\end{array}$ & $\begin{array}{l}5^{\prime}-\text { GACGTCCGGACTGGTTTGAT -3' } \\
5^{\prime}-\text { CACCGACACCCATACCAGAG -3' }\end{array}$ & $214 \mathrm{pb}$ & [36] \\
\hline HXT3 & $\begin{array}{l}\text { Forward } \\
\text { Reverse }\end{array}$ & $\begin{array}{l}\text { 5'-TTCGTTCCAGAATCCCCACG-3' }^{\prime} \\
\text { 5'-GAATGGATGGTCTGGGGCAA-3' }^{\prime}\end{array}$ & $102 \mathrm{bp}$ & [36] \\
\hline HXT4 & $\begin{array}{l}\text { Forward } \\
\text { Reverse }\end{array}$ & $\begin{array}{l}\text { 5'-CGCAGACGATCCAGCTGTTA-3' } \\
\text { 5'-CATGGAAGCAGCACCCCATA-3' }\end{array}$ & $343 \mathrm{bp}$ & [36] \\
\hline HXT6 & $\begin{array}{l}\text { Forward } \\
\text { Reverse }\end{array}$ & $\begin{array}{l}5^{\prime} \text {-GGTGAAGGTGAAGAGCACGA-3' } \\
5^{\prime} \text {-TCAAACCCACCTTACGACCG-3' }\end{array}$ & $313 \mathrm{bp}$ & [36] \\
\hline$H X T 7$ & $\begin{array}{l}\text { Forward } \\
\text { Reverse }\end{array}$ & $\begin{array}{l}5^{\prime}-\text { GGTGAAGGTGAAGAGCACGA-3' } \\
5^{\prime} \text {-TCAAACCCACCTTACGACCG-3's' }\end{array}$ & $313 \mathrm{bp}$ & [36] \\
\hline UBC6 & $\begin{array}{l}\text { Forward } \\
\text { Reverse }\end{array}$ & $\begin{array}{l}5^{\prime}- \\
\text { GATACTTGGAATCCTGGCTGGTCTGTCTC } \\
-3^{\prime} \\
5^{\prime}- \\
\text { AAAGGGTCTTCTGTTTCATCACCTGTATT } \\
\text { TGC-3 }\end{array}$ & $272 \mathrm{pb}$ & [37] \\
\hline
\end{tabular}

Table 2. Summary of phenotypes showed by BY4741, snfl $\triangle, h x k 2 \Delta$, and $h x k 2 \triangle / / G A L 1-H X K 2$ at $0.005 \%$ glucose

\begin{tabular}{|l|l|l|l|l|}
\hline Strain & $\begin{array}{l}\text { Specific } \\
\text { growth rate } \\
\left(\mathrm{h}^{-1}\right)\end{array}$ & $\begin{array}{l}\text { Basal genes } \\
\text { respiration } \\
(\text { nat O / } /\end{array}$ & $\begin{array}{l}\text { HXT } \\
\text { transcription } \\
\text { parameters }\end{array}$ \\
\hline
\end{tabular}




\begin{tabular}{|c|c|c|c|c|}
\hline & & $\begin{array}{l}\min x \mathrm{mg} \\
\text { of cell) }\end{array}$ & & \\
\hline BY4741 & $\begin{array}{l}0.08159 \pm \\
0.004226\end{array}$ & $\begin{array}{l}496.5 \pm \\
22.15\end{array}$ & $H X T 2(+)$ & $\begin{array}{l}\mathrm{Km} \sim 1.5 \\
\mathrm{mM} \\
\operatorname{Vmax} \sim 97 \\
\mathrm{nmol} / \mathrm{min} \\
\mathrm{mg}\end{array}$ \\
\hline snfl $\triangle$ & $\begin{array}{l}0.1798 \pm \\
0.01405\end{array}$ & $\begin{array}{l}1135 \pm \\
79.72\end{array}$ & $\begin{array}{l}H X T 3(+) \\
H X T 4(+)\end{array}$ & 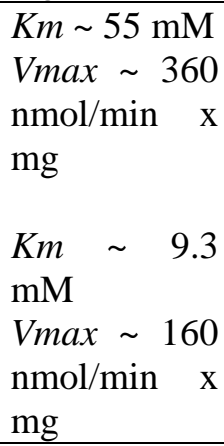 \\
\hline$h x k 2 \triangle$ & $\begin{array}{l}0.09928 \\
\pm \\
0.006587\end{array}$ & $\begin{array}{l}458.2 \pm \\
48.70\end{array}$ & $H X T 1(-)$ & $\begin{array}{l}K m \sim 90 \mathrm{mM} \\
V \max \sim 690 \\
\mathrm{nmol} / \mathrm{min} \quad \mathrm{x} \\
\mathrm{mg}\end{array}$ \\
\hline $\begin{array}{l}h x k 2 \triangle / / G A L 1- \\
H X K 2\end{array}$ & $\begin{array}{l}0.05681 \pm \\
0.002927\end{array}$ & $\begin{array}{l}427.1 \pm \\
66.38\end{array}$ & HXT1 (-) & $\begin{array}{l}K m \sim 90 \mathrm{mM} \\
\operatorname{Vmax} \sim 690 \\
\mathrm{nmol} / \mathrm{min} \quad \mathrm{x} \\
\mathrm{mg}\end{array}$ \\
\hline
\end{tabular}

Table 3. Summary of phenotypes showed by BY4741, snfl $\triangle, h x k 2 \triangle$, and $h x k 2 \triangle / / G A L 1-H X K 2$ at

\begin{tabular}{|c|c|c|c|c|}
\hline Strain & $\begin{array}{l}\text { Specific } \\
\text { growth rate } \\
\left(h^{-1}\right)\end{array}$ & $\begin{array}{l}\text { Basal } \\
\text { respiration } \\
\text { (nat O / } \\
\text { min x mg } \\
\text { of cell) }\end{array}$ & $\begin{array}{l}H X T \quad \text { genes } \\
\text { transcription }\end{array}$ & $\begin{array}{l}\text { Biochemical } \\
\text { parameters }\end{array}$ \\
\hline BY4741 & $\begin{array}{l}0.3669 \pm \\
0.009735\end{array}$ & $\begin{array}{l}276.5 \pm \\
7.415\end{array}$ & $\begin{array}{l}\text { HXT1 (+) } \\
\text { HХT3 (+) }\end{array}$ & 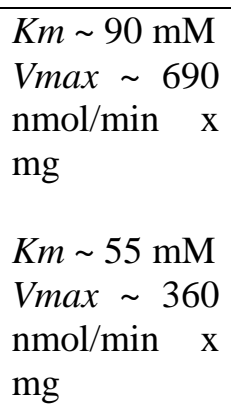 \\
\hline$s n f 1 \triangle$ & $\begin{array}{l}0.3528 \pm \\
0.01120\end{array}$ & $\begin{array}{l}251.7 \pm \\
28.18\end{array}$ & $\begin{array}{l}\text { HXTl (+) } \\
\text { HXT4 (+) }\end{array}$ & $\begin{array}{l}\text { Km } 90 \mathrm{mM} \\
\operatorname{Vmax} \sim 690 \\
\mathrm{nmol} / \mathrm{min} \quad \mathrm{x} \\
\mathrm{mg} \\
\begin{array}{l}\mathrm{Km} \sim 9.3 \\
\mathrm{mM}\end{array}\end{array}$ \\
\hline
\end{tabular}




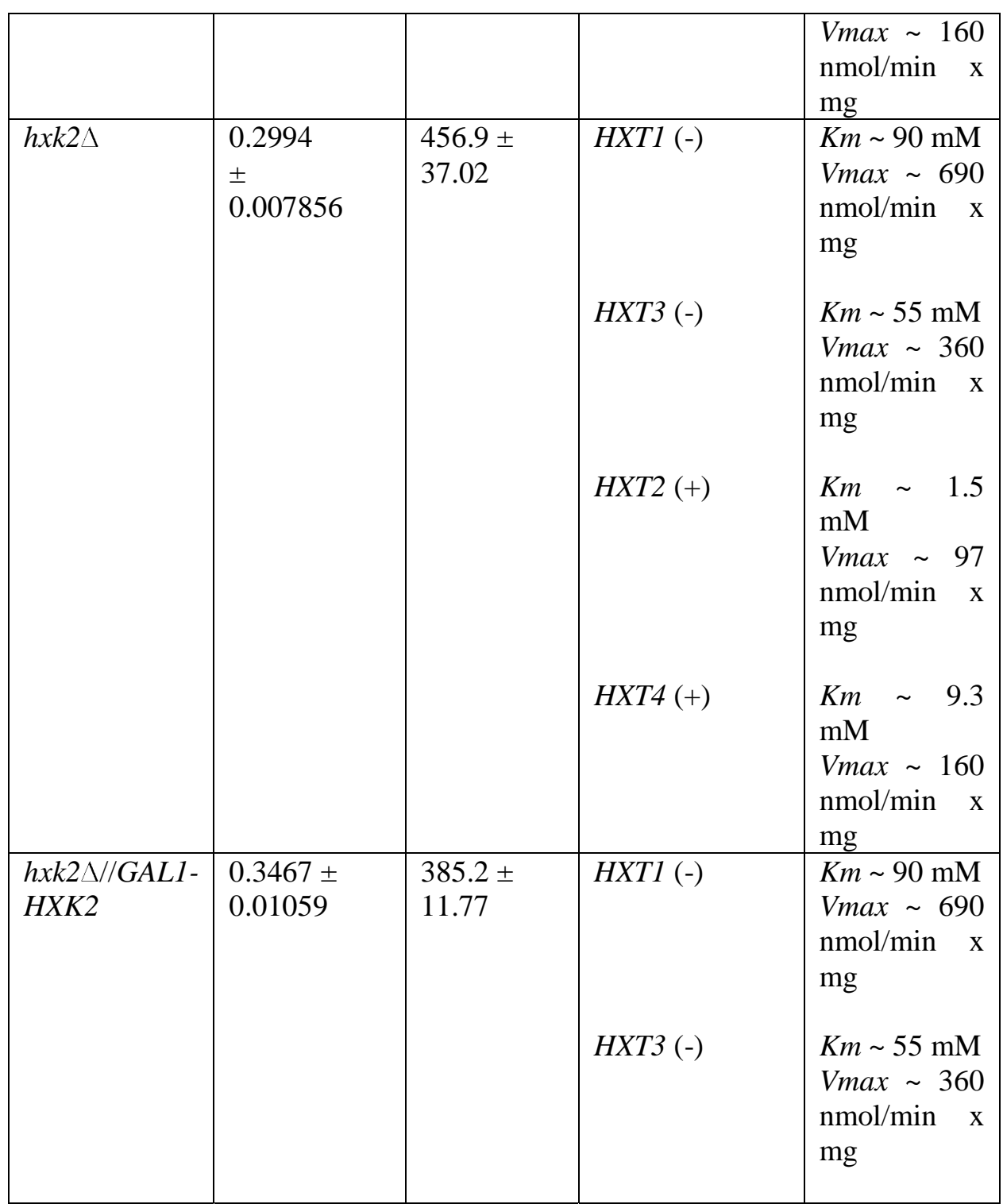

894

895

896

Table 4. Summary of phenotypes showed by BY4742 and snfl at $0.01 \%$ glucose

\begin{tabular}{|c|c|c|c|}
\hline Strain & $\begin{array}{l}\text { Specific } \\
\text { growth rate } \\
\left(h^{-1}\right)\end{array}$ & $\begin{array}{l}H X T \text { gene } \\
\text { transcription } \\
\text { augmented }\end{array}$ & $\begin{array}{l}\text { Biochemical } \\
\text { parameter } \\
\text { of } H X T \text { most } \\
\text { transcribed }\end{array}$ \\
\hline BY4742 & $\begin{array}{l}0.1118 \pm \\
0.008450\end{array}$ & $H X T 2(+)$ & $\begin{array}{l}\text { Km } \sim 1.5 \\
\mathrm{mM} \\
\operatorname{Vmax} \sim 97 \\
\mathrm{nmol} / \mathrm{min} \\
\mathrm{mg}\end{array}$ \\
\hline snfl $\triangle$ & $\begin{array}{l}0.02867 \\
\pm \\
0.005741\end{array}$ & $H X T 7(+)$ & $\begin{array}{l}\mathrm{Km} \sim 1.1 \\
\mathrm{mM} \\
\operatorname{Vmax} \sim 186 \\
\mathrm{nmol} / \min \mathrm{x}\end{array}$ \\
\hline
\end{tabular}




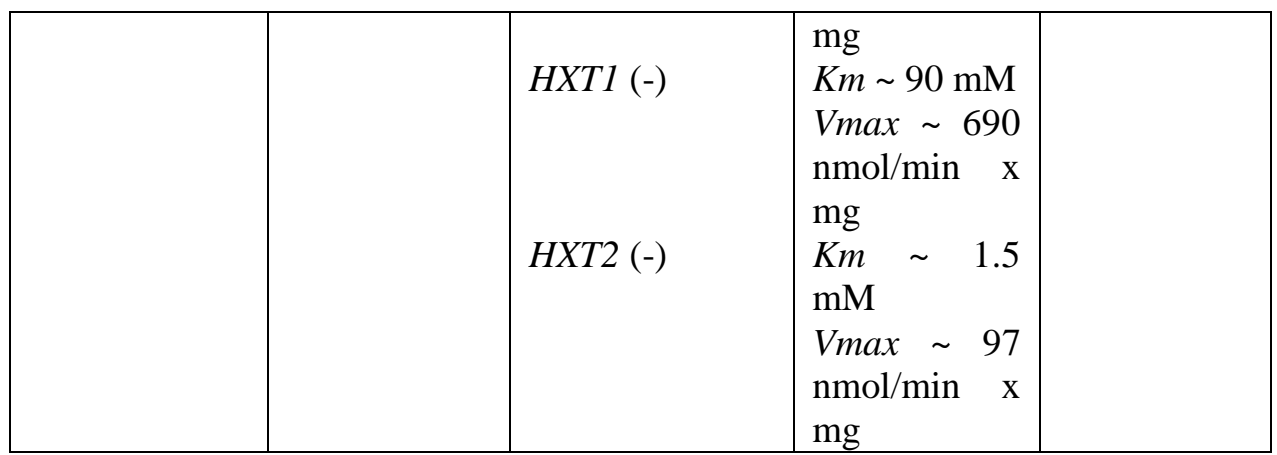

898 Table 5. Summary of phenotypes showed by BY4742 and snf1 at 5\% glucose 899

\begin{tabular}{|c|c|c|c|c|}
\hline Strain & $\begin{array}{l}\text { Specific } \\
\text { growth rate } \\
\left(h^{-1}\right)\end{array}$ & $\begin{array}{l}H X T \text { gene } \\
\text { transcription } \\
\text { augmented }\end{array}$ & $\begin{array}{l}\text { Biochemical } \\
\text { parameter } \\
\text { of } H X T \text { most } \\
\text { transcribed }\end{array}$ & 900 \\
\hline BY4742 & $\begin{array}{l}0.3255 \pm \\
0.008632\end{array}$ & $\begin{array}{l}\text { HXT1 (+) } \\
\text { HXT3 (+) }\end{array}$ & $\begin{array}{l}K m \sim 90 \mathrm{mM} \\
\operatorname{Vmax} \sim 690 \\
\mathrm{nmol} / \mathrm{min} \quad \mathrm{x} \\
\mathrm{mg} \\
\mathrm{Km} \sim 55 \mathrm{mM} \\
\operatorname{Vmax} \sim 360 \\
\mathrm{nmol} / \mathrm{min} \mathrm{x} \\
\mathrm{mg}\end{array}$ & \\
\hline snfl $\triangle$ & $\begin{array}{l}0.3433 \\
\pm \\
0.01653\end{array}$ & $\begin{array}{l}\text { HXT2 (-) } \\
\text { HXT6 (-) } \\
\text { HXT7 (-) }\end{array}$ & 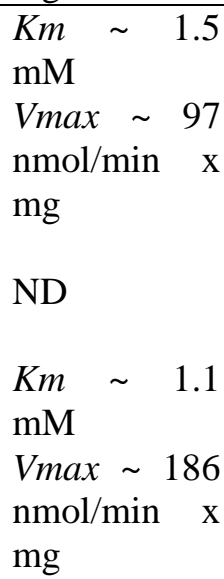 & \\
\hline
\end{tabular}


a)

Wild type (BY4741)

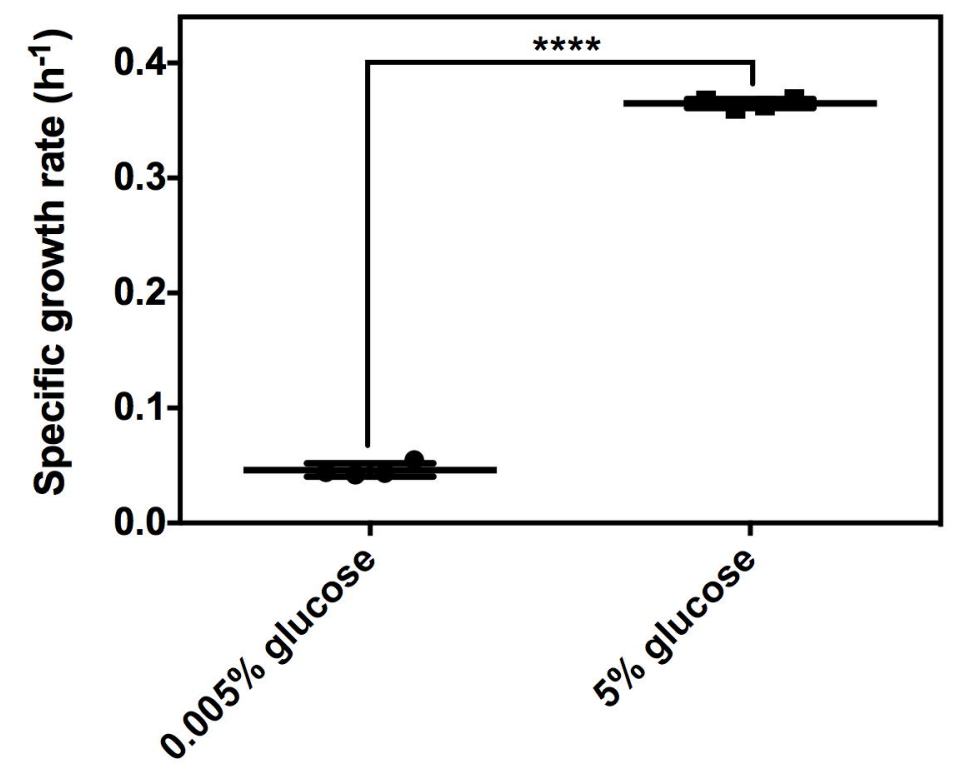

b)

$0.005 \%$ glucose

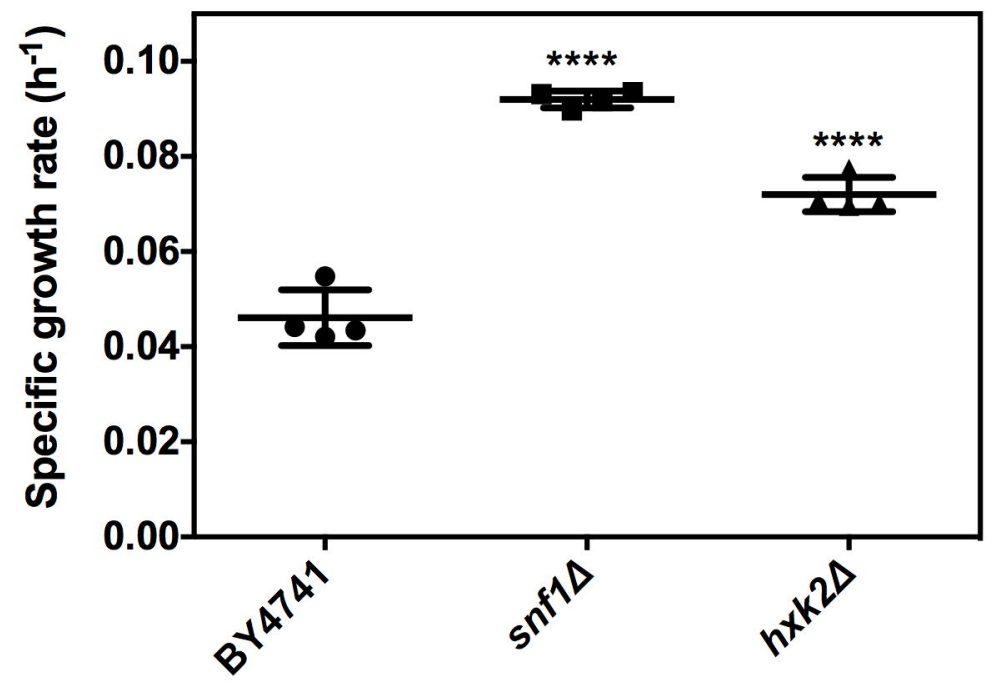

c)

$5 \%$ glucose

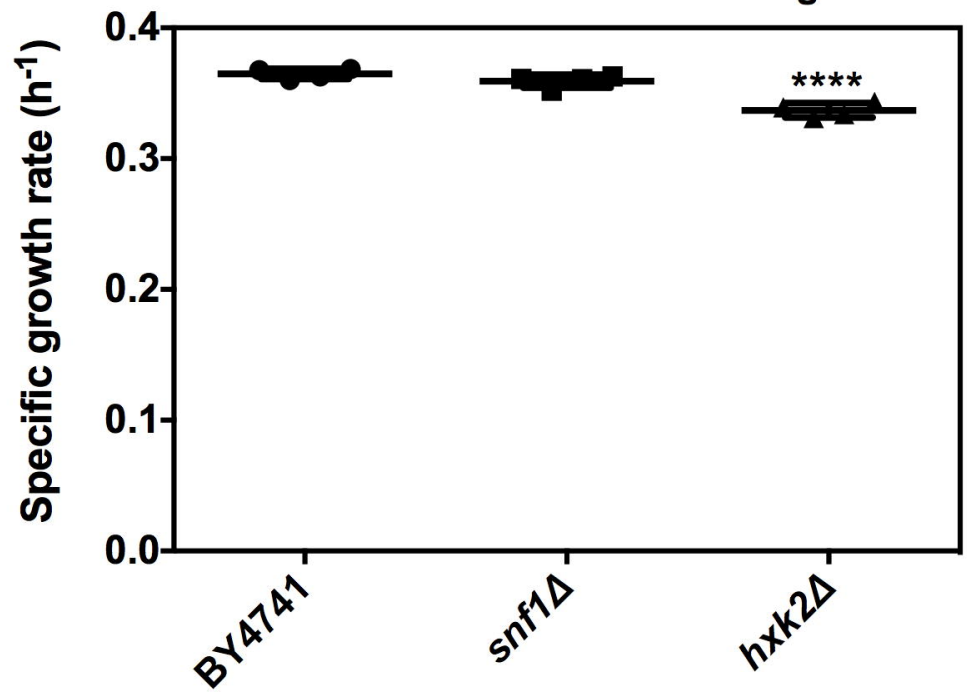




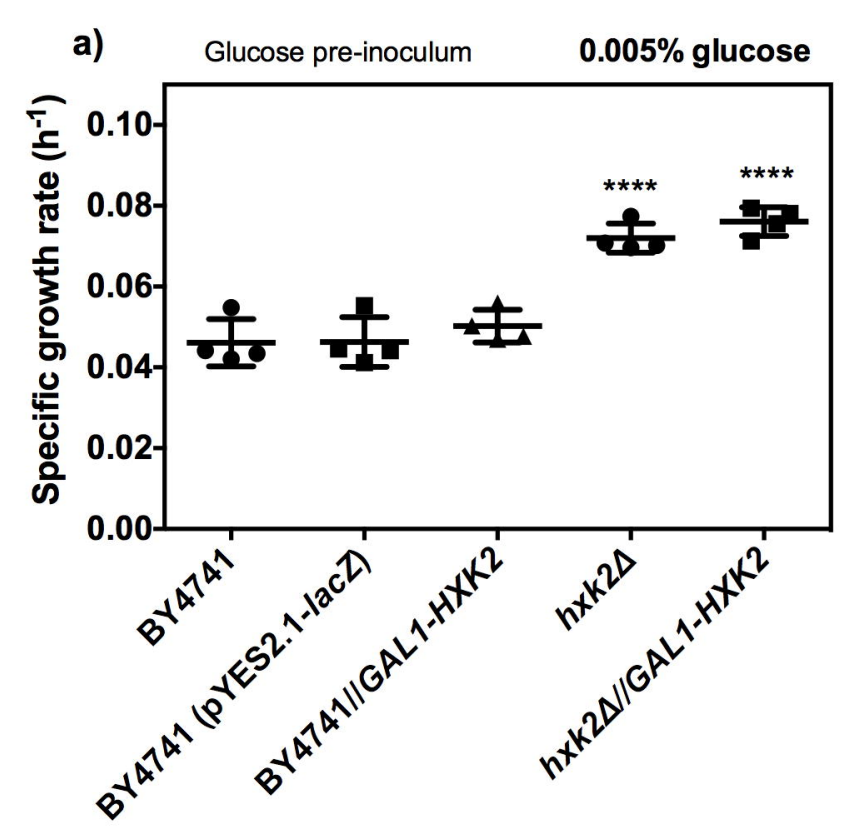

b) Glucose pre-inoculum $\quad 5 \%$ glucose
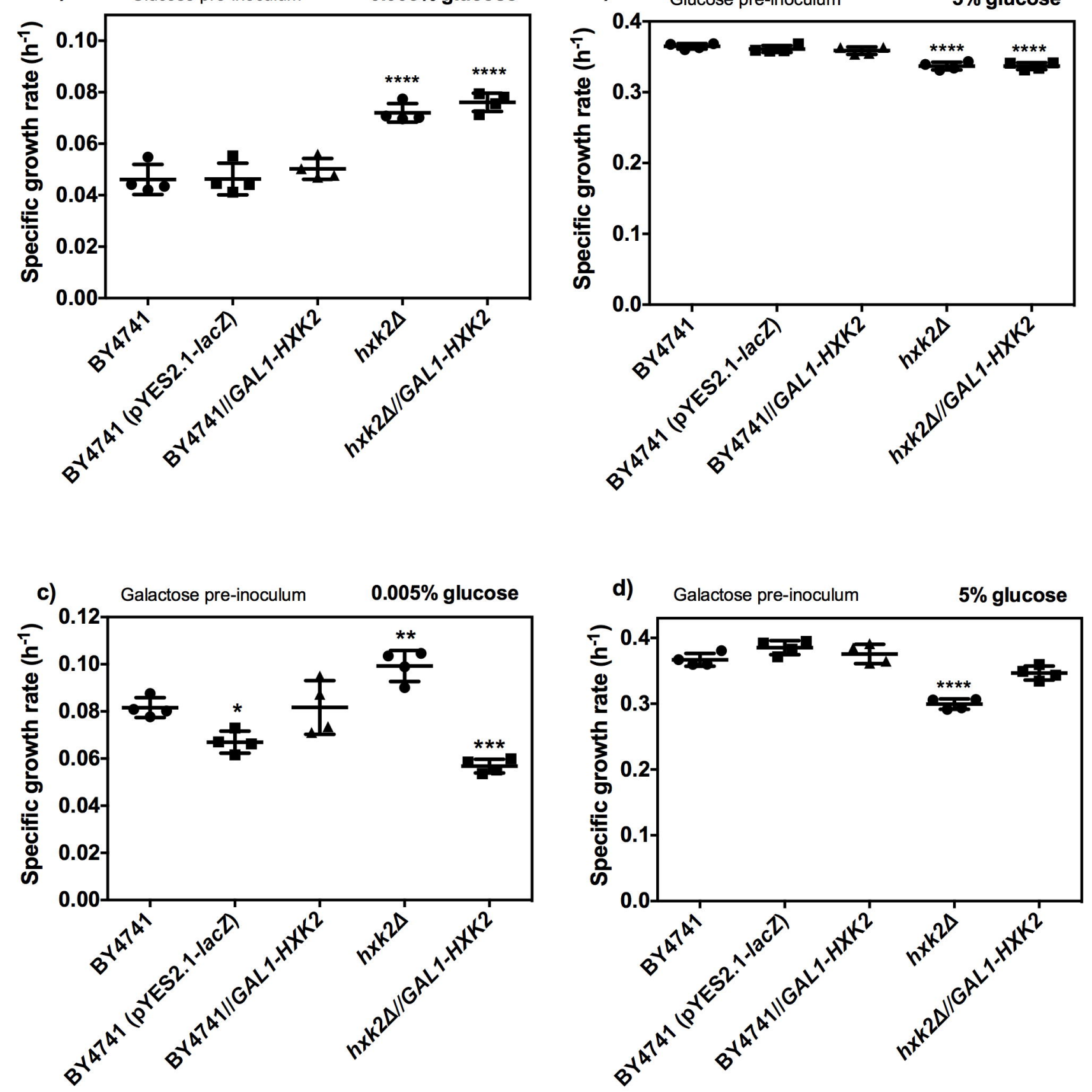

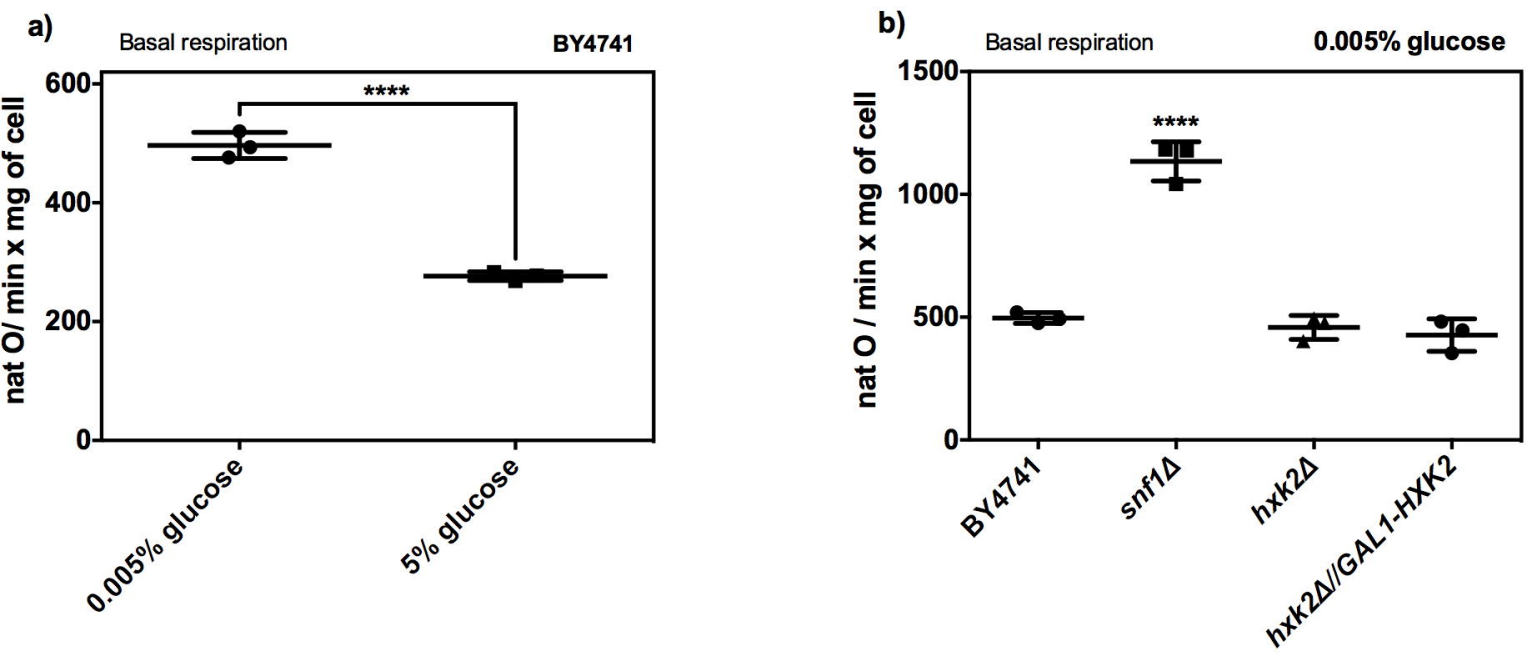

c)

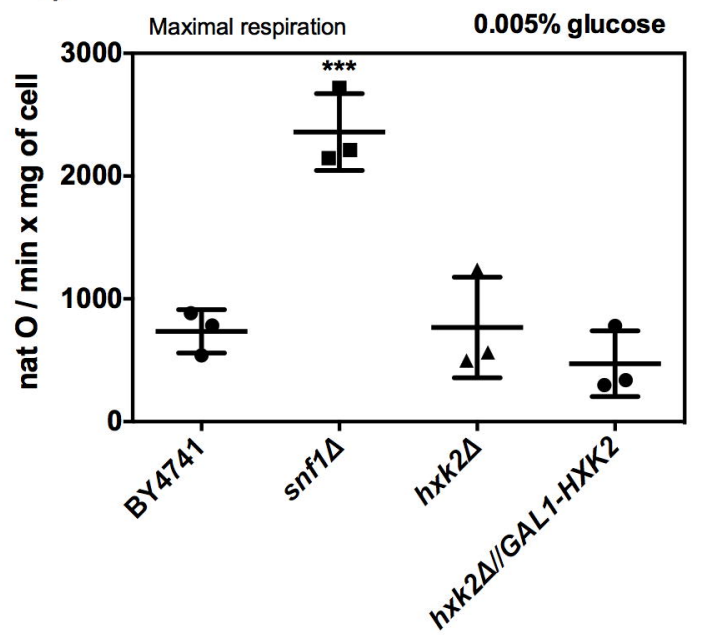

e)

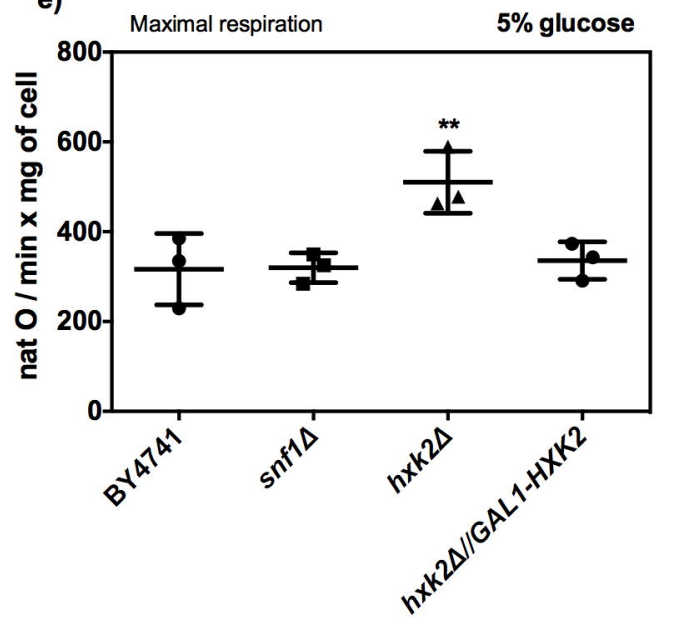

d)

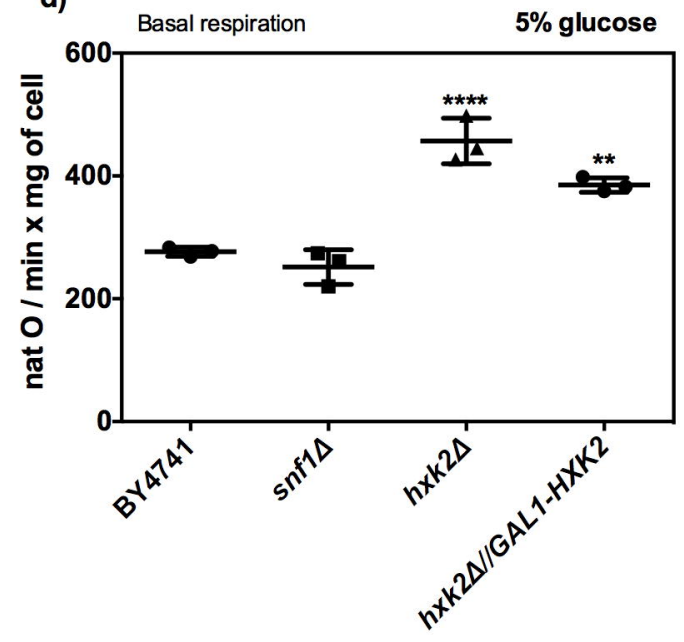

f)

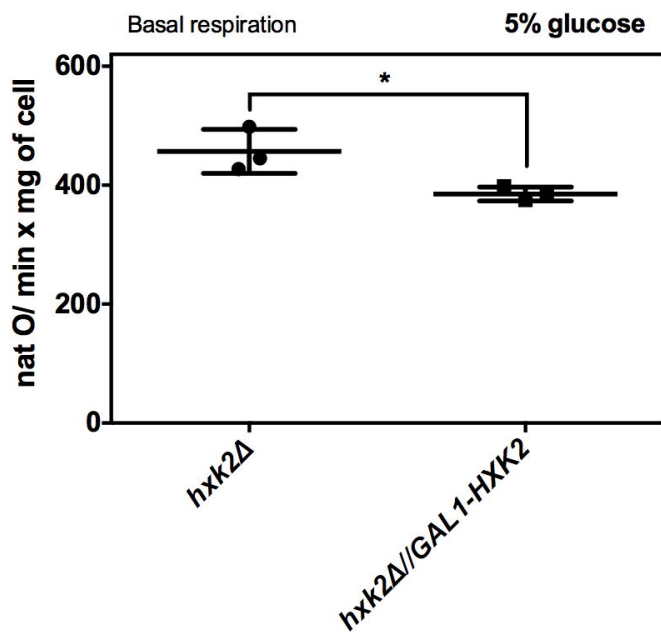




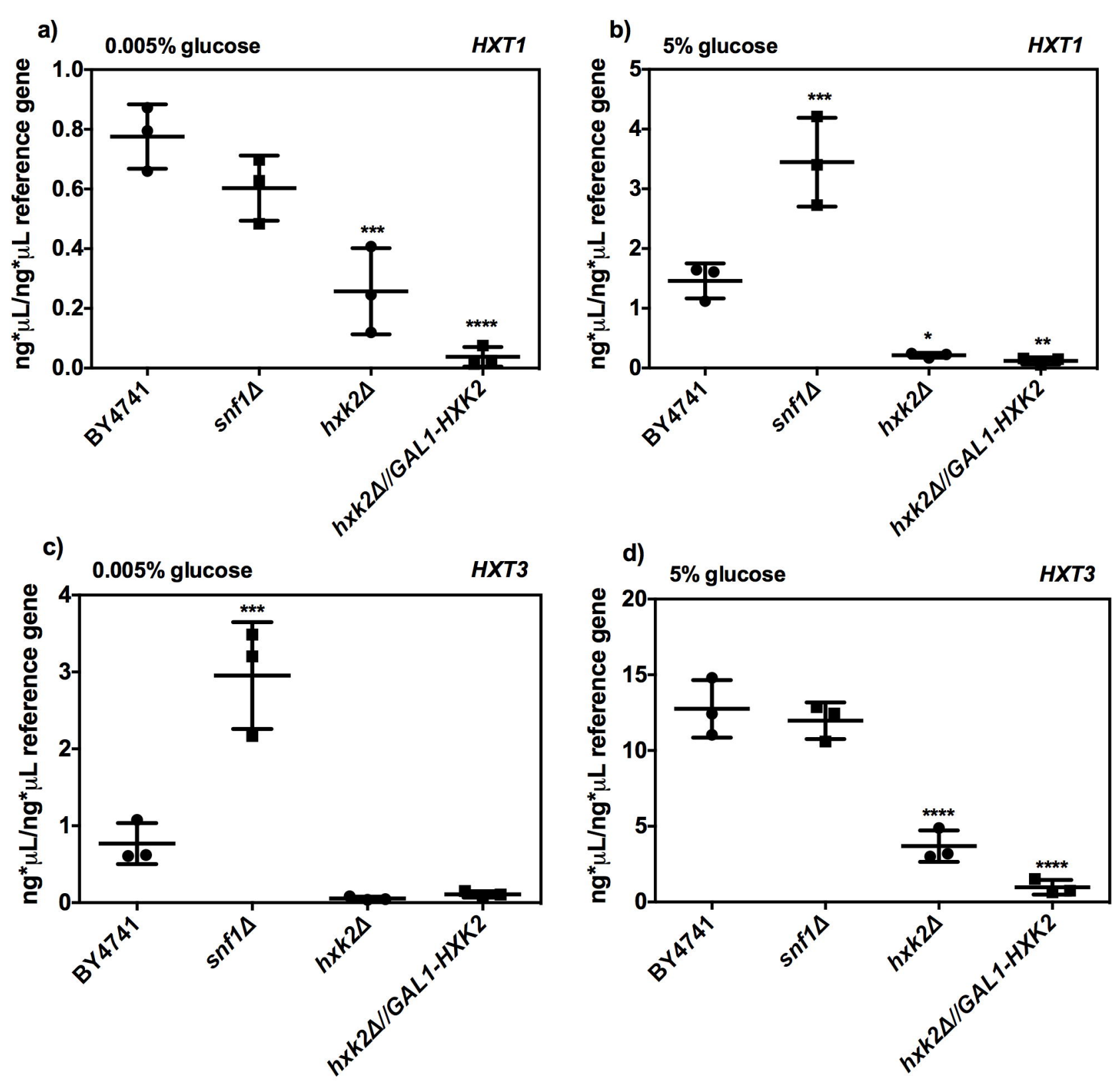



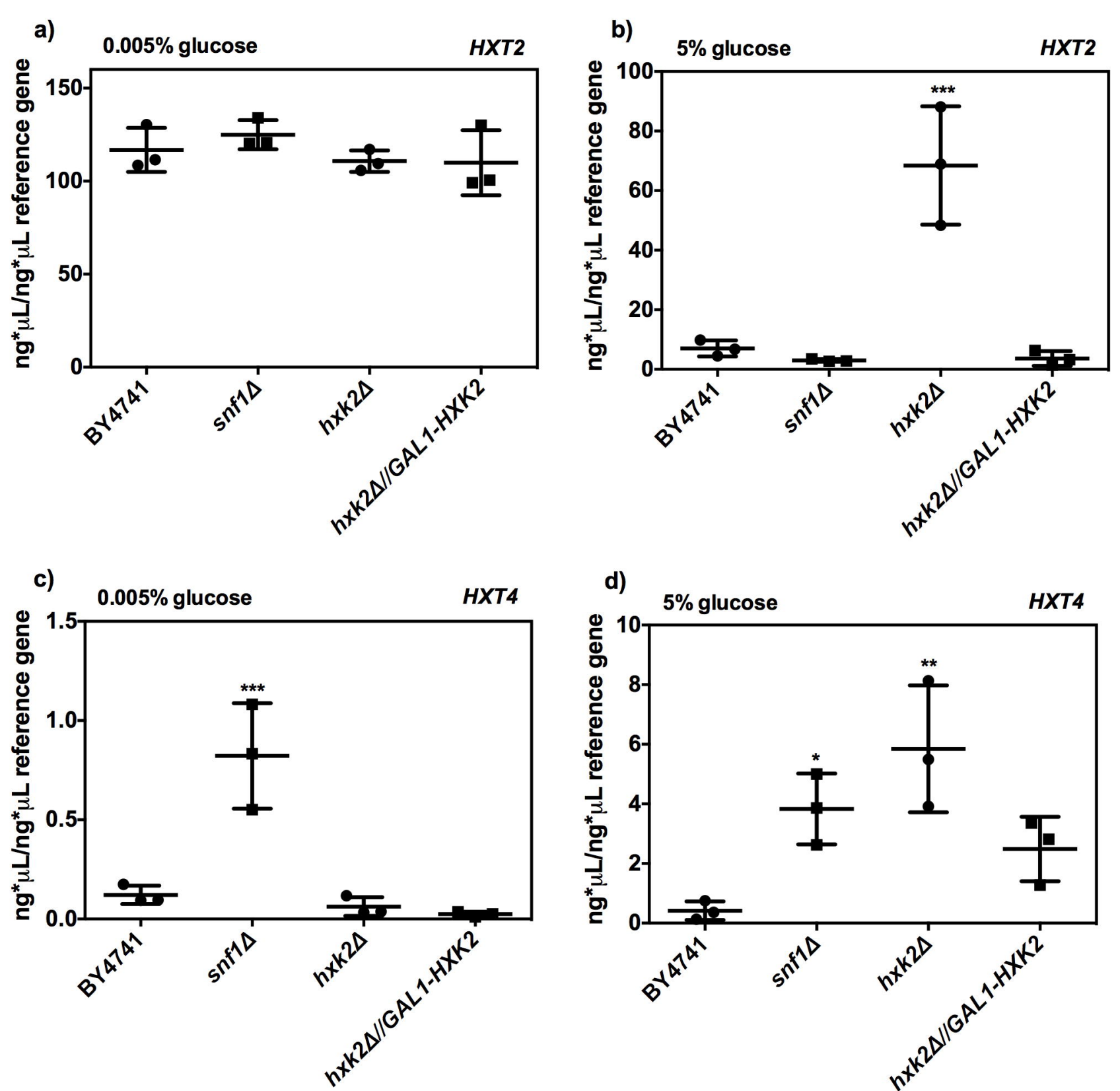

d)

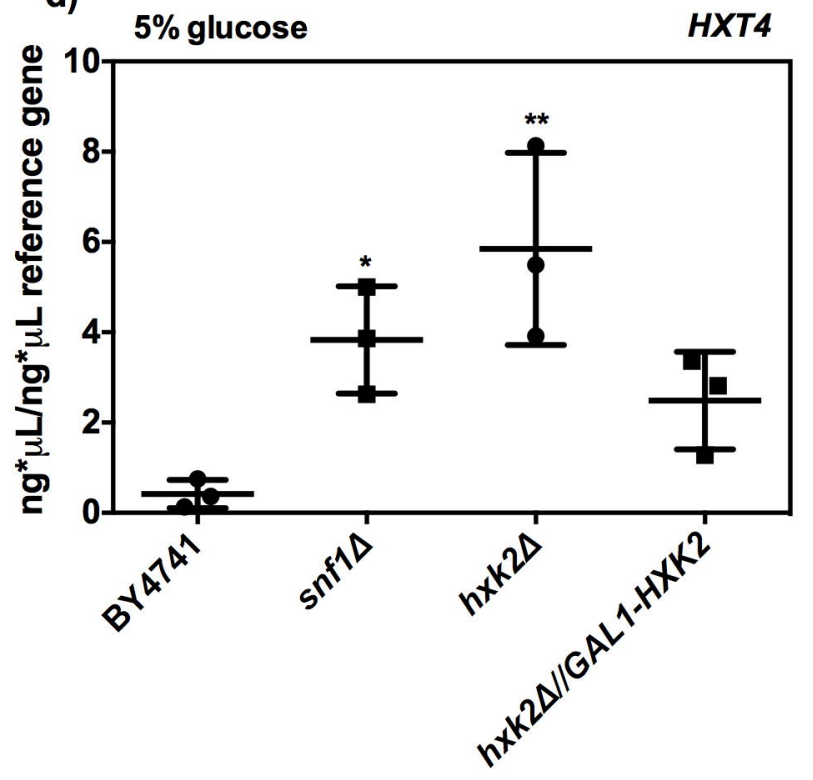


a)

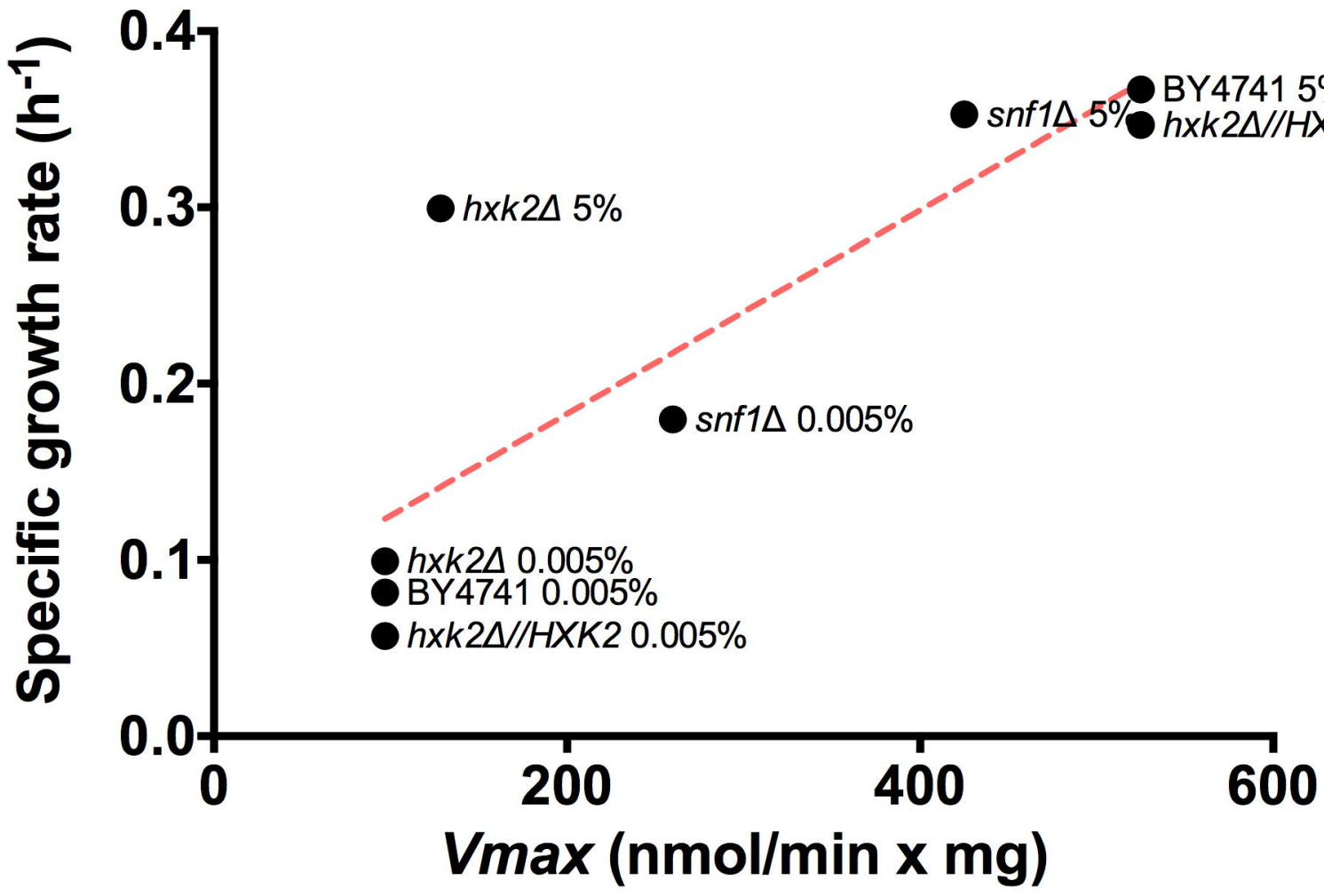

b)

600

400

BY4741 0.005\%

- $h$ K Kaxk2010\%

hxk2 $2 / / H X K 20.005 \%$

200

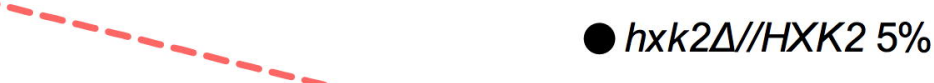

snf $1 \Delta 5 \%$ BY $47415 \%$

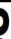

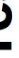


a)

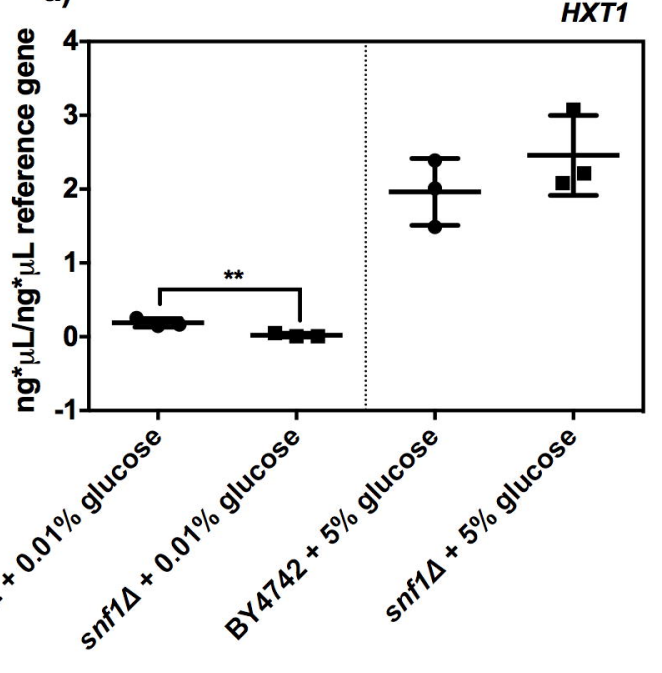

c)

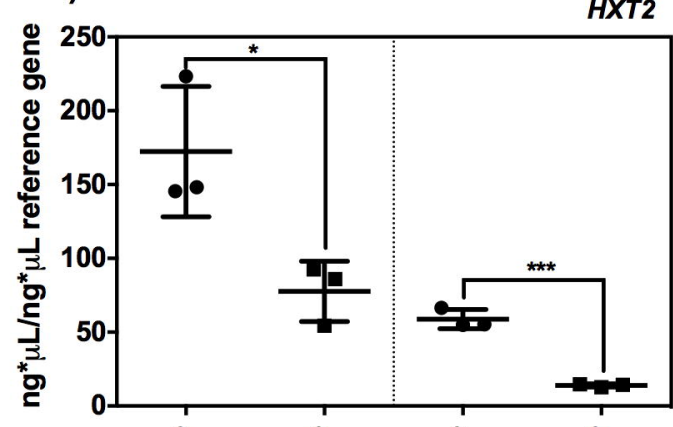

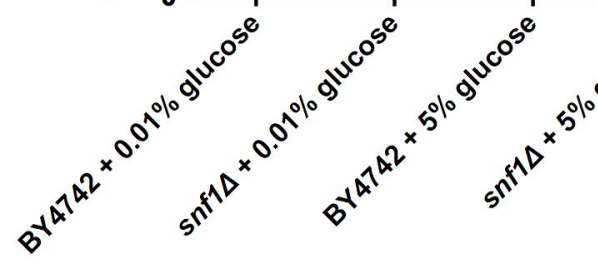

e)

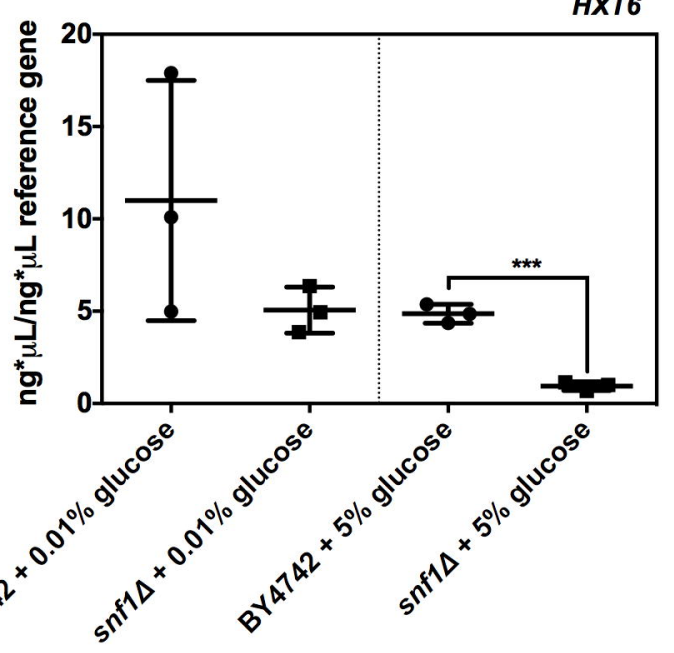

b)

HXT3

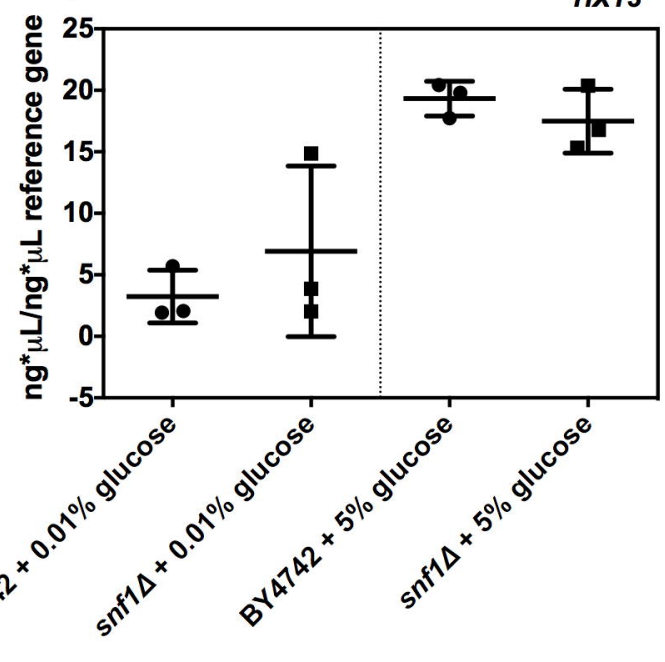

d)

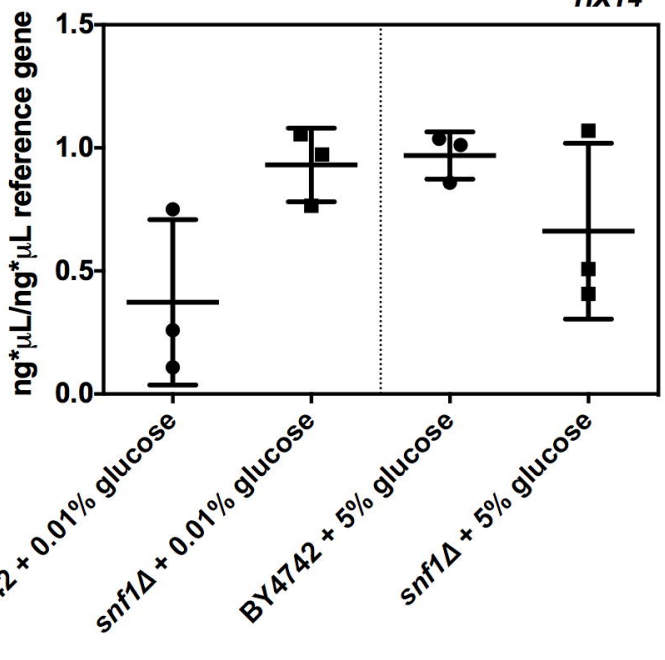

f)

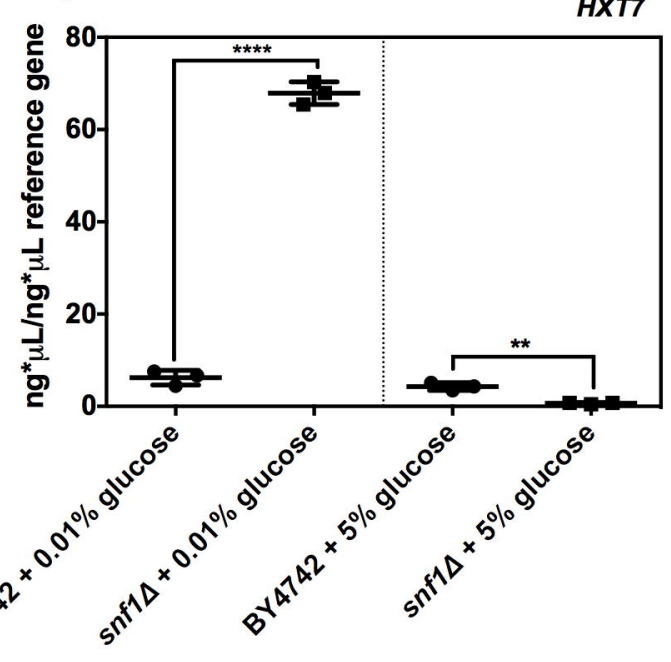




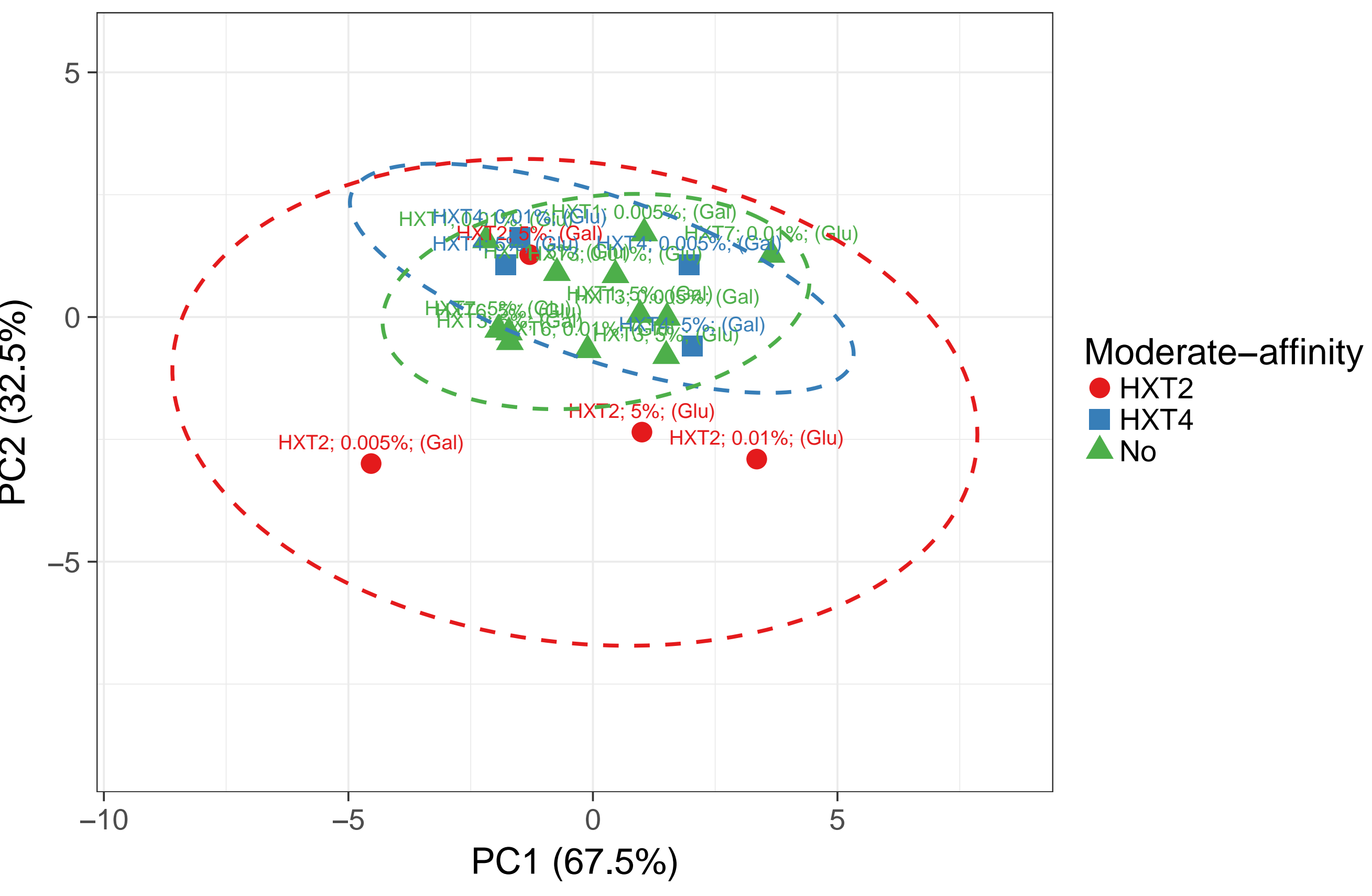


HXT2

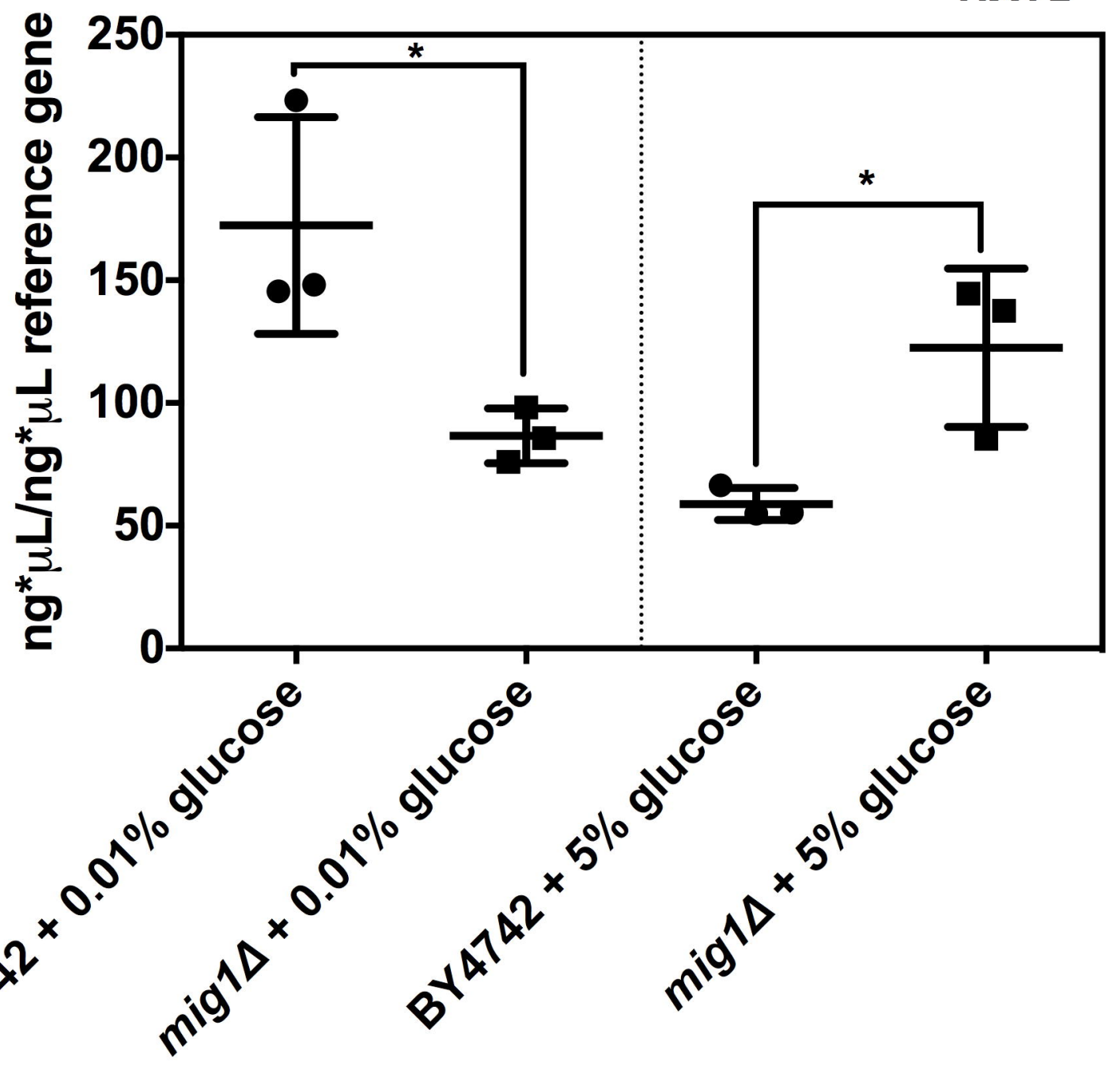


a)

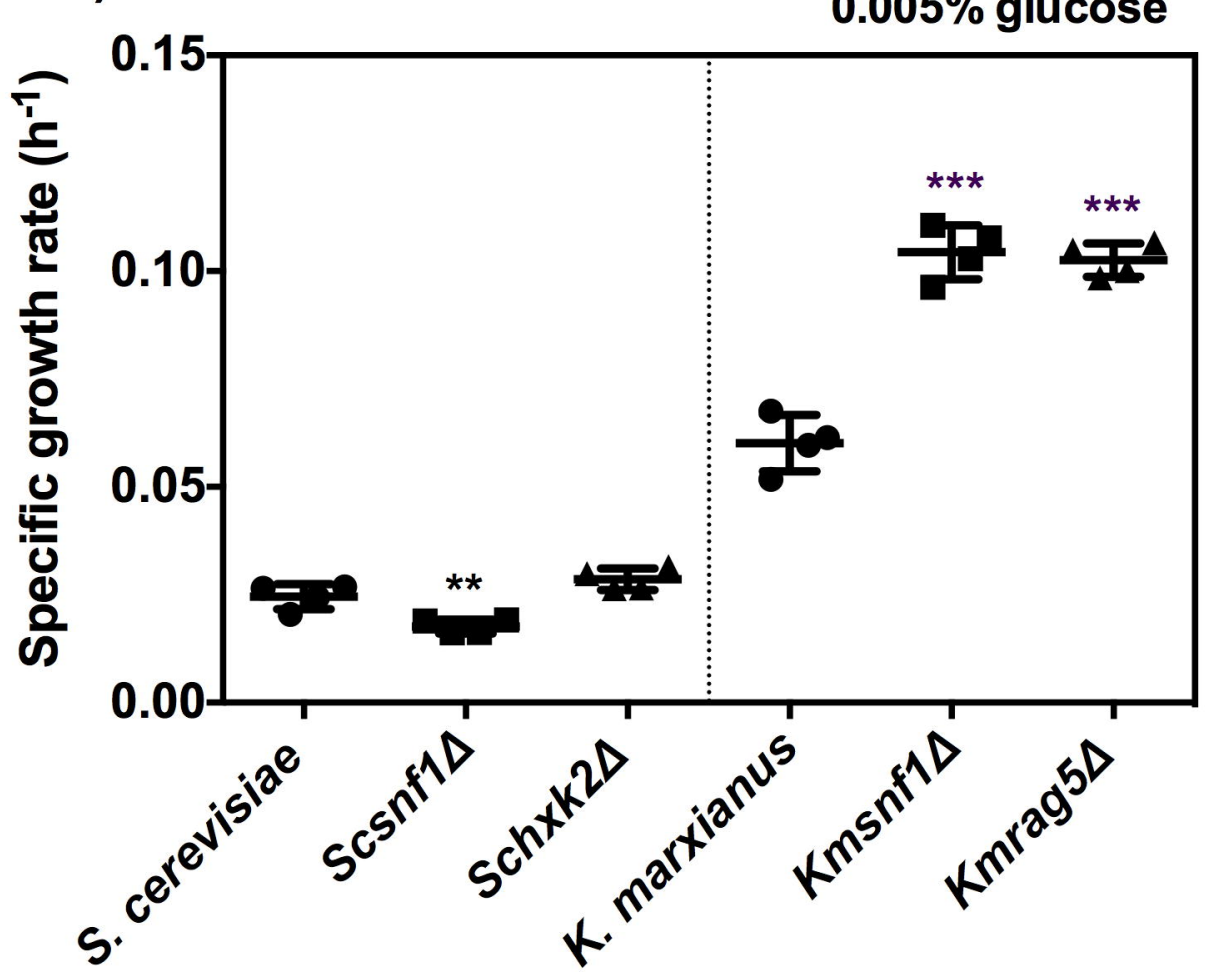

b)

$5 \%$ glucose

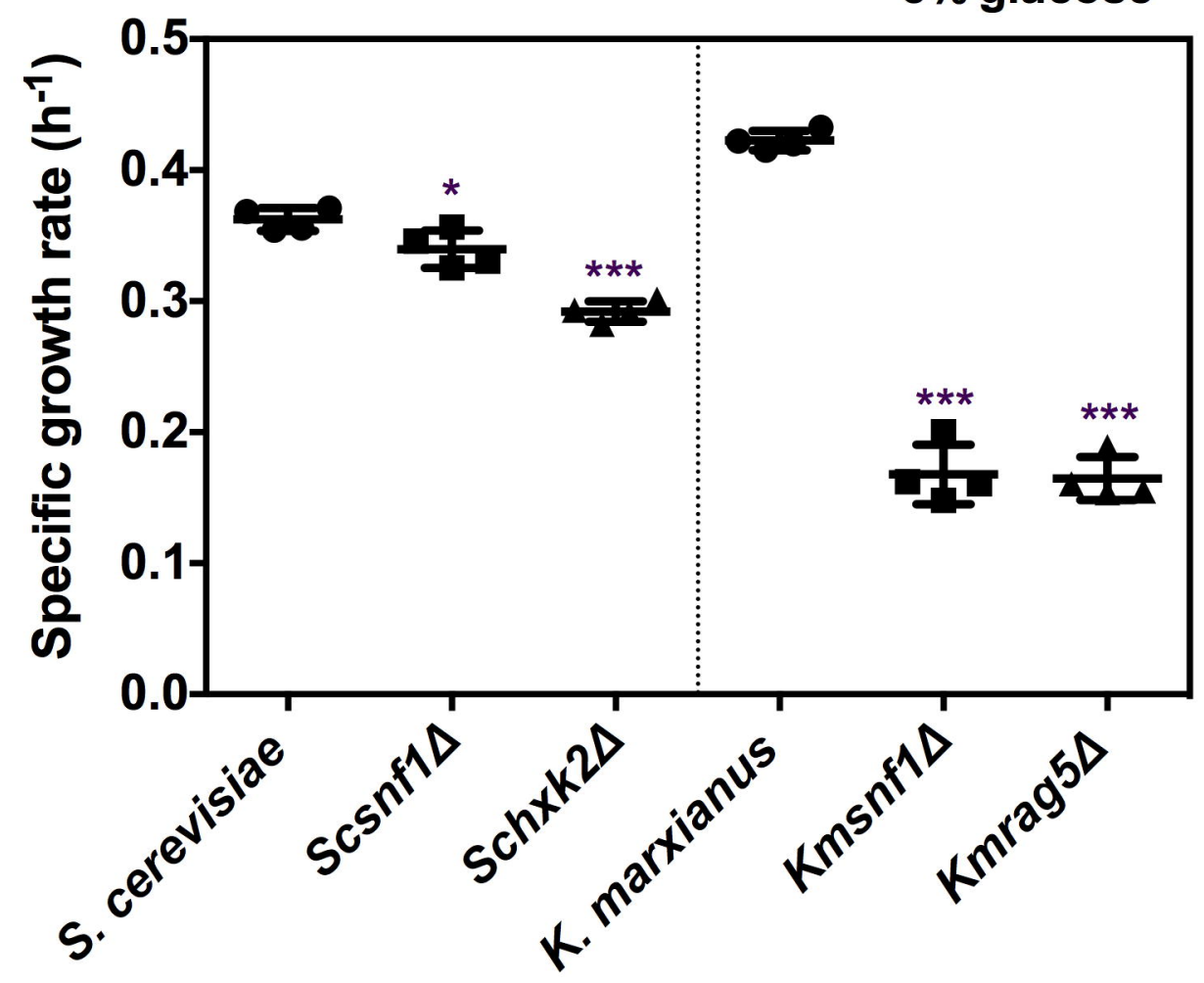


a)

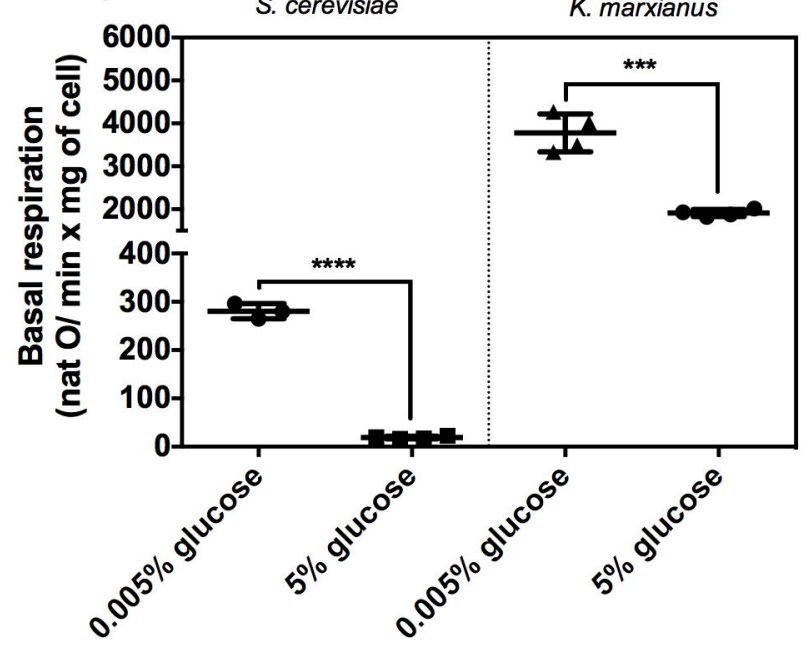

b)

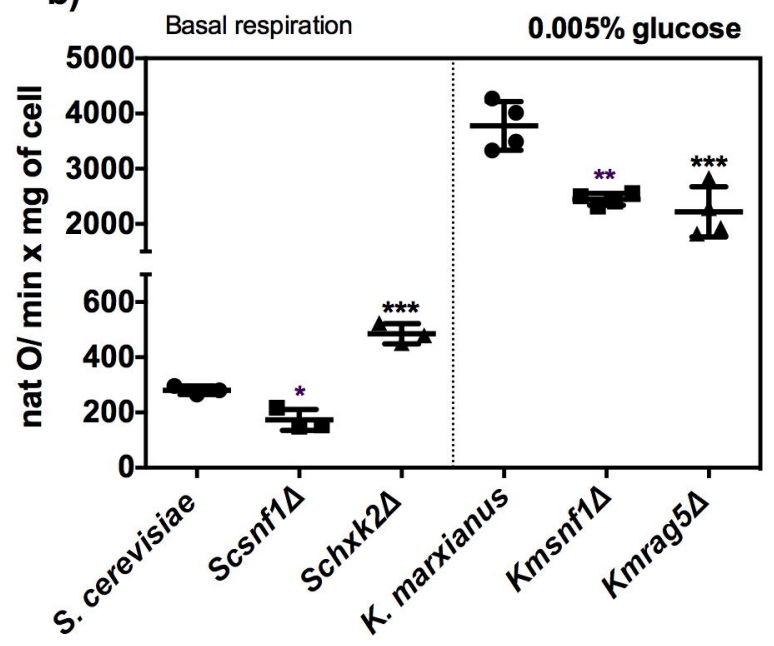

d)

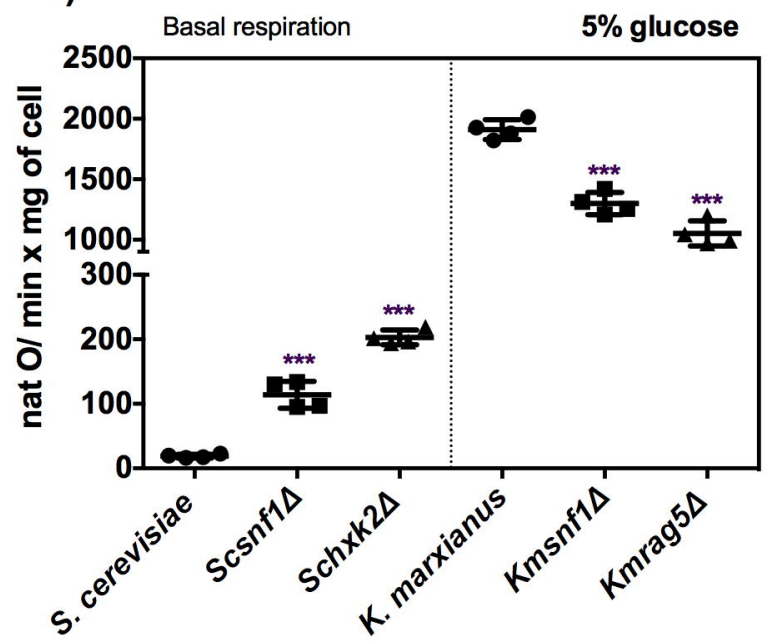

c)

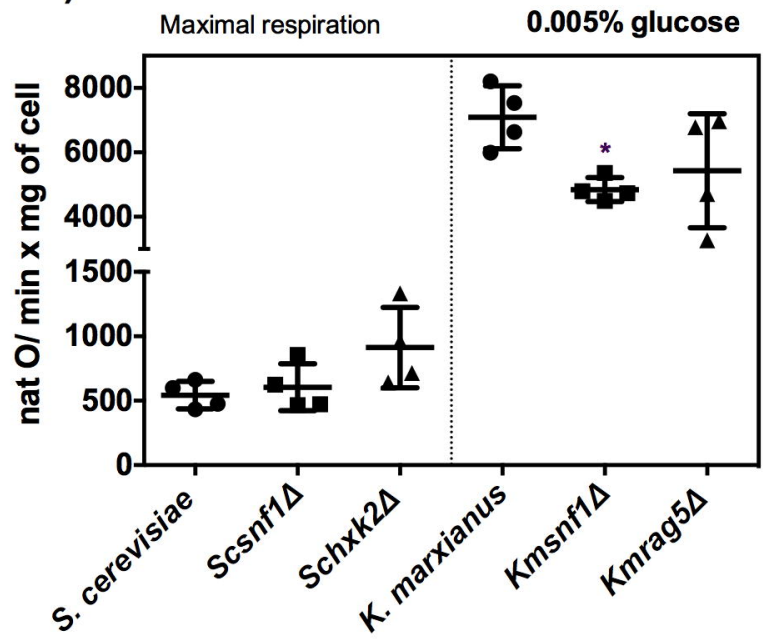

e)

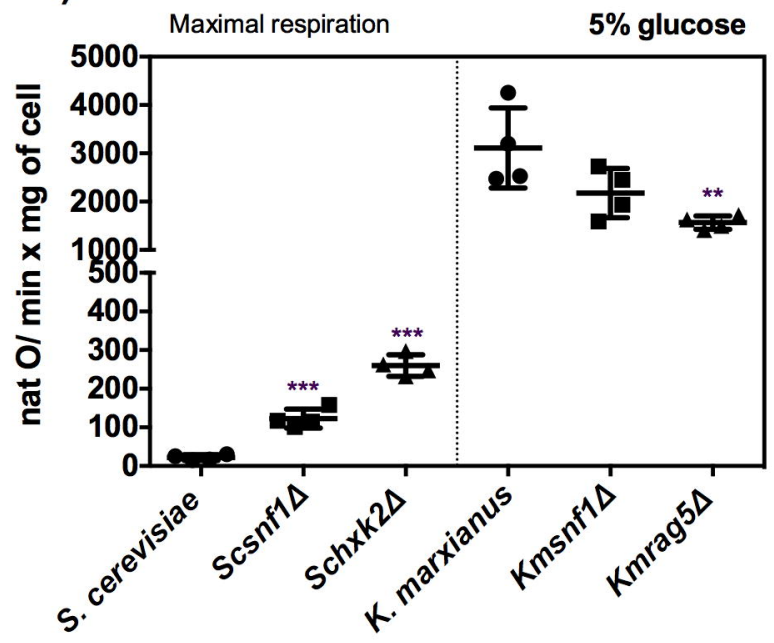

